\title{
THE ATKINSON-WILCOX THEOREM IN THERMOELASTICITY
}

\author{
BY \\ FIORALBA CAKONI (Department of Mathematics, University of Tirana, Albania) \\ AND
}

GEORGE DASSIOS (Division of Applied Math., Dept. of Chemical Engineering, University of Patras and ICEHT-FORTH, Greece)

\begin{abstract}
An incident disturbance propagates in a thermoelastic medium of the Biot type and it is scattered by a bounded discontinuity of the medium. On the surface of the scatterer any kind of boundary or transmission conditions, that secures well posedness, can hold. The scattered field consists of three kinds of displacement and two kinds of thermal waves. With the exception of one of the displacement waves, namely the transverse elastic wave, all other four scattered waves exhibit exponential attenuation as a result of the coupling between the longitudinal elastic and the thermal disturbances. We show that the displacement field can be expanded in three uniformly and absolutely convergent series in inverse powers of the distance between the observation point and the geometrical center of the scatterer. For the thermal wave a corresponding expansion with two series holds true. Each one of these three elastic and two thermal series describes the corresponding scattered wave and their validity is extended up to the sphere that circumscribes the scatterer. The leading coefficients in the two displacement series of the longitudinal type have only radial components which coincide with the corresponding radial scattering amplitudes. For the transverse displacement series the leading coefficient has only tangential components which coincide with the angular scattering amplitudes. An amazing result, which was not noticed before, is that the thermal scattering amplitudes, appearing as leading coefficients in the thermal expansions, are proportional to the corresponding radial longitudinal amplitudes of the elastic expansions. In other words, both scattering amplitudes of the two thermal waves carry no independent information about the scattering process. Finally, an analytic algorithm is provided which leads to the reconstruction of all five series from the knowledge of the three leading coefficients coming from the expansions for the displacement field alone. Consequently, if the radial and the tangential scattering amplitudes of the displacement field are given in the far field, then the exact displacement and thermal fields can be recovered all the way down to the smallest sphere containing the scatterer. In an equivalent component form we
\end{abstract}

Received January 27, 1998.

1991 Mathematics Subject Classification. Primary 35B40, 35C10, 35K20, 35L20, 73B30, 73D25. 
claim that the nine elastic and the two thermal expansions can be completely obtained once the two longitudinal and the two transverse elastic scattering amplitudes are given.

1. Introduction. In the first page of his monumental survey article [13] on the contributions of Rayleigh to scattering theory, Victor Twersky states: "So much of Rayleigh's work is "scattering theory" in the general sense the term is used today, and so much of it has been the basis for subsequent applications and extensions, that "Rayleigh" and "scattering" are practically synonymous". We are among those that can detect no exaggeration in such a statement. Indeed, the mathematical theory of scattering of waves by obstacles was formulated by Rayleigh in a series of over one hundred papers in the fifty years extent between 1870 and 1920 .

Rayleigh introduced an asymptotic form for the scattered field at infinity. He assumed that in the far field the scattered wave arrives in the form of a point-generated field with an amplitude that is dependent on the geometrical and physical characteristics of the scatterer. This is Rayleigh's radiation condition [13] and Rayleigh's scattering amplitude, which were introduced on the basis of physical arguments. A crucial instance in the development of scattering theory was the 1912 Sommerfeld's paper [12], where the behaviour of the scattered field at infinity was imposed by an asymptotic condition, which was dictated by the mathematical demands for well posedness and it was compatible with physical reality. A second important instance of scattering theory, less recognized but of great value, especially in inverse scattering theory, occurred in 1949 when Atkinson published his work "On Sommerfeld's Radiation Condition" [1]. Atkinson proved that the asymptotic condition of Sommerfeld can be replaced by a uniformly and absolutely convergent series representation of the scattered wave outside the smallest sphere that contains the scattering obstacle. This is an expansion in inverse powers of the radial distance and its leading term recovers Sommerfeld's Radiation Condition. The importance of Atkinson's work was pointed out by Wilcox [14] seven years later when he proved that once the leading coefficient of the series is known, all other coefficients are obtained through a recurrence relation. Since the leading coefficient is the scattering amplitude this means that, if the scattering amplitude is measured in the far field, then the exact scattered wave is recoverable up to the circumscribing of the scatterer sphere. This is an impressive inverse scattering result.

Atkinson's theorem provides the wave analogue of Maxwell's multipole expansion [11] in potential theory. The important difference between the two expansions is the following. In Maxwell's expansion the multipole moments that appear as coefficients of the expansion are independent, while in Atkinson's expansion all coefficients can be recovered from the leading one. This difference reflects the dynamic structure of the wave field as it compares to the static character of the potential.

Wilcox [15] extended Atkinson's theorem for electromagnetic scattering in 1959, where he proved that a corresponding reconstruction of the full series from the leading coefficient of the electric field was also possible. For Maxwell's equations, the algorithm of reconstruction was much more complicated than the scalar case of acoustical scattering. A further extension to elasticity was obtained by one of the authors in 1988 [4], where one can find a detailed historical guide on radiation conditions and exterior expansion 
theorems. For elastic wave scattering the expansion of the scattered wave involves two series, one for the longitudinal and one for the transverse wave. As was expected, the main difficulty was focused on the development of the analytical algorithm that recovers all the coefficients of the expansions in terms of the leading ones. An appropriate decomposition of the coefficients into radial and tangential spherical components confirmed such a reconstruction. It was proved that the expansion of the longitudinal scattered wave is recoverable from the radial scattering amplitude alone, and that the same is true for the transverse scattered wave, where the corresponding expansion is recoverable only from the tangential scattering amplitude. As far as relative expansion theorems in two dimensions are concerned the only contribution known to the authors is Karp's work [9].

The theory of thermoelastic scattering was developed in a series of papers $[3,5,6$, $7,8]$ by Kostopoulos and the authors. Thermoelasticity couples the hyperbolic character of elastic wave propagation with the parabolic behaviour of heat conduction. As a result of this coupling, thermal waves are generated and, at the same time, the elastic waves exhibit exponential attenuation. Hence, the effects that one field has on the other are not symmetric and this is reflected upon the lack of selfadjointness of the governing thermoelastic operator. The thermoelastic coupling gives rise to three elastic and two thermal waves. Two out of the three elastic waves are longitudinal pressure waves and they are coupled with the two scalar thermal waves. The third elastic wave is a transverse shear wave that is not affected by the thermal field since it has an equivoluminal character. We refer to the first longitudinal elastic wave as the elastothermal wave, since it reduces to the longitudinal wave of classical elasticity whenever the coupling parameters tend to zero. The second longitudinal elastic wave recovers the thermal field, as the system decouples, and it is known as a thermoelastic wave. As is expected from such a coupled system of equations, thermoelastic scattering problems involve a high level of mathematical complexity. In order to compactify some of this complexity a fourdimensional formulation has been proposed [5] which involves the three components of the displacement field plus the temperature field.

In this work we study the form that Atkinson's theorem assumes in the theory of thermoelasticity. The formulation of thermoelastic scattering is outlined in Sec. 2. In Sec. 3 we develop Atkinson's theorem for thermoelastic waves and in Sec. 4 we describe the algorithm that leads to the reconstruction of the full expansions (the leading coefficients of the three out of the five series are known). Equivalently, in component form, four scalar functions are enough to reconstruct in full the eleven scalar series involved in the expansion of the displacement and the temperature fields. This is a rather complicated and by no means straightforward mechanism that demands an appropriate resolution of interwoven sequences of coefficients. In any case, it is of interest to see here that the thermal far-field patterns are not needed to obtain the full expansion, since the scattering amplitudes associated with the temperature fields are proportional to the corresponding amplitudes associated with the longitudinal displacement fields. Finally, all previous forms of this expansion theorem in acoustics, electromagnetism, and elasticity are recovered in Sec. 5, as special cases of its thermoelastic version. The large amount of analytic calculations, at the dyadic and triadic level needed in this work, forced us to find a way to isolate and compactify the basic formulae that come from generic calculations. 
These are collected in the Appendix and they form a useful set of formulae for calculations with point sources and fundamental solutions in continuum mechanics.

2. Thermoelastic scattering. Consider a compact subset $V^{-}$of $\mathbb{R}^{3}$, with a smooth boundary $S$, which we will refer to as the scatterer. The complement $V$ of $V^{-}$provides the exterior medium of propagation and it is occupied by a linear isotropic and homogenous thermoelastic medium of the Biot type [2]. The Biot medium is characterized by the Lamé elastic constants $\lambda, \mu$, the constant mass density $\rho$, the coefficient of thermal diffusivity $\kappa$, and the two coupling constants $\gamma$ and $\eta$. Suppressing the harmonic time dependence $\exp \{-i \omega t\}$ with frequency $\omega$, the Biot system assumes the following spectral form:

$$
\begin{gathered}
\mu \Delta \mathbf{u}+(\lambda+\mu) \nabla \nabla \cdot \mathbf{u}+\rho \omega^{2} \mathbf{u}=\gamma \nabla \theta, \\
\Delta \theta+q \theta=-i \omega \eta \nabla \cdot \mathbf{u},
\end{gathered}
$$

where $\mathbf{u}$ is the elastic displacement field, $\theta$ denotes the temperature variation field, and

$$
q=\frac{i \omega}{\kappa}
$$

is a spectral thermal constant.

From (1) we observe that the temperature gradient acts as a source for the displacement field, while (2) implies that the sources for the temperature field are controlled by the divergence of the velocity field (the factor $-i \omega$ comes from the time derivative of $\mathbf{u}$ ). The system (1), (2) can be unified into a four-dimensional formulation with the help of the unified field

$$
\mathbf{U}(\mathbf{r})=\left(u_{1}(\mathbf{r}), u_{2}(\mathbf{r}), u_{3}(\mathbf{r}), \theta(\mathbf{r})\right)
$$

and the block matrix operator

$$
\tilde{\mathbf{L}}=\left[\begin{array}{c|c}
\left(\mu \Delta+\rho \omega^{2}\right) \widetilde{\mathbf{I}}_{3}+(\lambda+\mu) \nabla \nabla & -\gamma \nabla \\
\hline q \kappa \eta \nabla & \Delta+q
\end{array}\right]
$$

where $\widetilde{\mathbf{I}}_{3}$ is the identity dyadic in three dimensions. In fact, the system (1), (2) is now written as

$$
\widetilde{\mathbf{L}} \mathbf{U}=\mathbf{0}
$$

and the solution space is reduced to the kernel of $\widetilde{\mathbf{L}}$. The dispersion relations [5] characterizing (6) are given by

$$
\begin{aligned}
k_{1}^{2}+k_{2}^{2} & =q(1+\varepsilon)+k_{p}^{2}, \\
k_{1}^{2} k_{2}^{2} & =q k_{p}^{2}, \\
c_{s}^{2} k_{s}^{2} & =\omega^{2},
\end{aligned}
$$

where $k_{1}, k_{2}$ are the complex wavenumbers of the elastothermal and the thermoelastic wave, respectively, $k_{s}$ is the wavenumber of the uncoupled transverse wave, $k_{p}$ is the 
wavenumber of the longitudinal wave in the absence of thermal coupling, and

$$
\varepsilon=\frac{\gamma \eta \kappa}{\lambda+2 \mu}
$$

is the dimensionless thermoelastic coupling constant [10]. From (9) we see that the transverse elastic wave is not affected by the existence of the temperature field and it behaves exactly the same way as it does in the classical theory of elasticity. Consistency with physical reality demands that for $j=1,2$

$$
k_{j}=\frac{\omega}{v_{j}}+\mathrm{id}_{j}, \quad v_{j}>0, d_{j}>0
$$

where $v_{1}, v_{2}$ are the phase velocities and $d_{1}, d_{2}$ are the dissipation coefficients for the elastothermal and the thermoelastic waves respectively.

The unified incident plane wave that excites the scatterer assumes the general form $[5]$

$$
\widetilde{\mathbf{\Phi}}(\mathbf{r})=A^{1} e^{i k_{1} \hat{\mathbf{k}} \cdot \mathbf{r}}\left(\hat{\mathbf{k}}, \beta_{1}\right)+A^{2} e^{i k_{2} \hat{\mathbf{k}} \cdot \mathbf{r}}\left(\beta_{2} \hat{\mathbf{k}}, 1\right)+A^{s} e^{i k_{s} \hat{\mathbf{k}} \cdot \mathbf{r}}(\hat{\mathbf{b}}, 0)
$$

where $A^{1}, A^{2}, A^{s}$ indicate the amplitudes of the waves corresponding to the three wave numbers $k_{1}, k_{2}, k_{s}$, respectively, $\hat{\mathbf{k}}$ is the direction of propagation, $\hat{\mathbf{b}}$ is the polarization vector of the $S$-wave, which is perpendicular to $\hat{\mathbf{k}}$, and

$$
\begin{aligned}
& \beta_{1}=\frac{i k_{1} q \kappa \eta}{k_{1}^{2}-q} \\
& \beta_{2}=\frac{i k_{2} \gamma}{\rho c_{p}^{2}\left(k_{p}^{2}-k_{2}^{2}\right)}
\end{aligned}
$$

are the appropriate factors that force $\widetilde{\boldsymbol{\Phi}}$ to live in the kernel of $\widetilde{\mathbf{L}}$.

A set of radiation conditions at infinity and another set of boundary conditions on $S$ are prescribed in [5] which are not repeated here since they are not important for our expansion theorem. What is important is the following integral representation [5] of the scattered field $\mathbf{U}$ which incorporates the above conditions:

$$
\begin{aligned}
\mathbf{U}(\mathbf{r})=\frac{1}{4 \pi} \int_{S}\left[\mathbf{U}\left(\mathbf{r}^{\prime}\right)\right. & \cdot \widetilde{\mathbf{R}^{(*)}}\left(\partial_{\mathbf{r}^{\prime}}, \hat{\mathbf{n}}^{\prime}\right) \widetilde{\mathbb{E}}^{\top}\left(\mathbf{r}, \mathbf{r}^{\prime}\right) \\
& \left.-\widetilde{\mathbf{E}}\left(\mathbf{r}, \mathbf{r}^{\prime}\right) \cdot \widetilde{\mathbf{R}}\left(\partial_{\mathbf{r}^{\prime}}, \hat{\mathbf{n}}^{\prime}\right) \mathbf{U}\left(\mathbf{r}^{\prime}\right)\right] d s\left(\mathbf{r}^{\prime}\right)
\end{aligned}
$$

where "T" denotes transposition, the operator " $(*)$ " is defined by

$$
\widetilde{\mathbf{A}}^{(*)}(\gamma, q \kappa \eta)=\widetilde{\mathbf{A}}(q \kappa \eta, \gamma),
$$

and the thermoelastic surface traction operator $\widetilde{\mathbf{R}}$ assumes the form

$$
\tilde{\mathbf{R}}\left(\partial_{\mathbf{r}}, \hat{\mathbf{n}}\right)=\left[\begin{array}{c|c}
\widetilde{\mathbf{T}}\left(\partial_{\mathbf{r}}, \hat{\mathbf{n}}\right) & -\gamma \hat{\mathbf{n}} \\
\hline \mathbf{0} & \hat{\mathbf{n}} \cdot \nabla
\end{array}\right]
$$

with

$$
\widetilde{\mathbf{T}}\left(\partial_{\mathbf{r}}, \hat{\mathbf{n}}\right) \mathbf{u}(\mathbf{r})=2 \mu \hat{\mathbf{n}} \cdot \nabla \mathbf{u}(\mathbf{r})+\lambda \hat{\mathbf{n}} \nabla \cdot \mathbf{u}(\mathbf{r})+\mu \hat{\mathbf{n}} \times(\nabla \times \mathbf{u}(\mathbf{r}))
$$

being the elastic surface traction operator, and $\widetilde{\mathbf{E}}$ is the fundamental solution of the operator $\widetilde{\mathbf{L}}$. The form of $\widetilde{\mathbf{E}}$ is very important for our work since it is this particular 
function that carries the $\mathbf{r}$-dependence of $\mathbf{U}$ in the representation (15). The fundamental solution is expressed as

$$
\widetilde{\mathbf{E}}\left(\mathbf{r}, \mathbf{r}^{\prime}\right)=\widetilde{\mathbf{D}}^{1}\left(\partial_{\mathbf{r}}\right) \frac{e^{i k_{1}\left|\mathbf{r}-\mathbf{r}^{\prime}\right|}}{\left|\mathbf{r}-\mathbf{r}^{\prime}\right|}+\widetilde{\mathbf{D}}^{2}\left(\partial_{\mathbf{r}}\right) \frac{e^{i k_{2}\left|\mathbf{r}-\mathbf{r}^{\prime}\right|}}{\left|\mathbf{r}-\mathbf{r}^{\prime}\right|}+\widetilde{\mathbf{D}}^{s}\left(\partial_{\mathbf{r}}\right) \frac{e^{i k_{s}\left|\mathbf{r}-\mathbf{r}^{\prime}\right|}}{\left|\mathbf{r}-\mathbf{r}^{\prime}\right|}
$$

where

$$
\begin{aligned}
& \widetilde{\mathbf{D}}^{1}\left(\partial_{\mathbf{r}}\right)=\frac{-1}{\rho \omega^{2}\left(k_{1}^{2}-k_{2}^{2}\right)}\left[\begin{array}{c|c}
\left(k_{p}^{2}-k_{2}^{2}\right) \nabla_{\mathbf{r}} \nabla_{\mathbf{r}} & \gamma k_{p}^{2} \nabla_{\mathbf{r}} \\
\hline-q \kappa \eta k_{p}^{2} \nabla_{\mathbf{r}} & \rho \omega^{2}\left(k_{p}^{2}-k_{1}^{2}\right)
\end{array}\right], \\
& \widetilde{\mathbf{D}}^{2}\left(\partial_{\mathbf{r}}\right)=\frac{1}{\rho \omega^{2}\left(k_{1}^{2}-k_{2}^{2}\right)}\left[\begin{array}{c|c}
\left(k_{p}^{2}-k_{1}^{2}\right) \nabla_{\mathbf{r}} \nabla_{\mathbf{r}} & \gamma k_{p}^{2} \nabla_{\mathbf{r}} \\
\hline-q \kappa \eta k_{p}^{2} \nabla_{\mathbf{r}} & \rho \omega^{2}\left(k_{p}^{2}-k_{2}^{2}\right)
\end{array}\right], \\
& \widetilde{\mathbf{D}}^{s}\left(\partial_{\mathbf{r}}\right)=\frac{1}{\rho \omega^{2}}\left[\begin{array}{c|c}
k_{s}^{2} \widetilde{\mathbf{I}}_{3}+\nabla_{\mathbf{r}} \nabla_{\mathbf{r}} & \mathbf{0} \\
\hline \mathbf{0} & 0
\end{array}\right] .
\end{aligned}
$$

Representation (15) can be modified [5] to express the scattered field in terms of the total field

$$
\boldsymbol{\Psi}(\mathbf{r})=\boldsymbol{\Phi}(\mathbf{r})+\mathbf{U}(\mathbf{r})
$$

and the total surface traction field $\widetilde{\mathbf{R}} \boldsymbol{\Psi}$ in the form

$$
\begin{aligned}
\mathbf{U}(\mathbf{r})=\frac{1}{4 \pi} \int_{S}\left[\Psi\left(\mathbf{r}^{\prime}\right)\right. & \cdot \widetilde{\mathbf{R}}(*)\left(\partial_{\mathbf{r}^{\prime}}, \hat{\mathbf{n}}^{\prime}\right) \widetilde{\mathbf{E}}^{\top}\left(\mathbf{r}, \mathbf{r}^{\prime}\right) \\
& \left.-\widetilde{\mathbf{E}}\left(\mathbf{r}, \mathbf{r}^{\prime}\right) \cdot \widetilde{\mathbf{R}}\left(\partial_{\mathbf{r}^{\prime}}, \hat{\mathbf{n}}^{\prime}\right) \Psi\left(\mathbf{r}^{\prime}\right)\right] d s\left(\mathbf{r}^{\prime}\right),
\end{aligned}
$$

which is appropriate for application of boundary conditions.

Asymptotic analysis of (24) for $r \rightarrow \infty$ leads to the following far-field forms:

$$
\begin{aligned}
\mathbf{U}(r) & =\mathbf{U}^{1}(\mathbf{r})+\mathbf{U}^{2}(\mathbf{r})+\mathbf{U}^{s}(\mathbf{r}), \\
\mathbf{U}^{1}(\mathbf{r}) & =\mathbf{G}^{1}(\hat{\mathbf{r}}, \hat{\mathbf{k}}) e^{-d_{1} r} h\left(\frac{\omega}{v_{1}} r\right)+O\left(\frac{1}{r^{2} e^{d_{1} r}}\right), \quad r \rightarrow \infty, \\
\mathbf{U}^{2}(\mathbf{r}) & =\mathbf{G}^{2}(\hat{\mathbf{r}}, \hat{\mathbf{k}}) e^{-d_{2} r} h\left(\frac{\omega}{v_{2}} r\right)+O\left(\frac{1}{r^{2} e^{d_{2} r}}\right), \quad r \rightarrow \infty, \\
\mathbf{U}^{s}(\mathbf{r}) & =\mathbf{G}^{s}(\hat{\mathbf{r}}, \hat{\mathbf{k}}) h\left(\frac{\omega}{c_{s}} r\right)+O\left(\frac{1}{r^{2}}\right), \quad r \rightarrow \infty,
\end{aligned}
$$

where the scattering amplitudes $\mathbf{G}^{1}, \mathbf{G}^{2}, \mathbf{G}^{s}$ are given in [5]. Their exact form, which is rather complicated, is not important for the present work. Decomposing the asymptotic 
expressions (25)-(28) into their elastic and thermal parts we arrive at

$$
\begin{aligned}
\mathbf{u}(\mathbf{r}) & =\mathbf{u}^{1}(\mathbf{r})+\mathbf{u}^{2}(\mathbf{r})+\mathbf{u}^{s}(\mathbf{r}), \\
\mathbf{u}^{1}(\mathbf{r}) & =g_{r}^{1}(\hat{\mathbf{r}}, \hat{\mathbf{k}}) e^{-d_{1} r} h\left(\frac{\omega}{v_{1}} r\right) \hat{\mathbf{r}}+O\left(\frac{1}{r^{2} e^{d_{1} r}}\right), \quad r \rightarrow \infty, \\
\mathbf{u}^{2}(\mathbf{r}) & =g_{r}^{2}(\hat{\mathbf{r}}, \hat{\mathbf{k}}) e^{-d_{2} r} h\left(\frac{\omega}{v_{2}} r\right) \hat{\mathbf{r}}+O\left(\frac{1}{r^{2} e^{d_{2} r}}\right), \quad r \rightarrow \infty, \\
\mathbf{u}^{s}(\mathbf{r}) & =\left[g_{\theta}^{s}(\hat{\mathbf{r}}, \hat{\mathbf{k}}) \hat{\boldsymbol{\theta}}+g_{\phi}^{s}(\hat{\mathbf{r}}, \hat{\mathbf{k}}) \hat{\boldsymbol{\varphi}}\right] h\left(\frac{\omega}{c_{s}} r\right)+O\left(\frac{1}{r^{2}}\right), \quad r \rightarrow \infty,
\end{aligned}
$$

for the three types of displacement fields, and

$$
\begin{aligned}
\theta(\mathbf{r}) & =\theta^{1}(\mathbf{r})+\theta^{2}(\mathbf{r}) \\
\theta^{1}(\mathbf{r}) & =\ell^{1}(\hat{\mathbf{r}}, \hat{\mathbf{k}}) e^{-d_{1} r} h\left(\frac{\omega}{v_{1}} r\right)+O\left(\frac{1}{r^{2} e^{d_{1} r}}\right), \quad r \rightarrow \infty \\
\theta^{2}(\mathbf{r}) & =\ell^{2}(\hat{\mathbf{r}}, \hat{\mathbf{k}}) e^{-d_{2} r} h\left(\frac{\omega}{v_{2}} r\right)+O\left(\frac{1}{r^{2} e^{d_{2} r}}\right), \quad r \rightarrow \infty
\end{aligned}
$$

for the two types of temperature fields.

The elastic scattering amplitudes corresponding to the $\mathbf{u}^{1}$ and $\mathbf{u}^{2}$ fields are radial functions expressing the longitudinal character of these fields, while the $\mathbf{u}^{s}$ field generates a tangential scattering amplitude that establishes its transverse character. Besides the geometrical attenuation described by the Hankel function $h$, the waves $\mathbf{u}^{1}, \mathbf{u}^{2}, \theta^{1}$, and $\theta^{2}$ exhibit also the physical dissipation expressed by the exponentials $\exp \left\{-d_{i} r\right\}, i=1,2$. On the other hand, the field $\mathbf{u}^{s}$, which is not coupled with $\theta$, exhibits no such dissipation. Complete expressions for $g_{r}^{1}, g_{r}^{2}, g_{\theta}^{s}, g_{\phi}^{s}, \ell^{1}$, and $\ell^{2}$ can be found in [5].

3. The expansion theorem. In this section we develop the expansion theorem of Atkinson, starting form the representation (24). Our first task is to apply all the differentiations on $\widetilde{\mathbf{E}}$ involved in the operators $\widetilde{\mathbf{D}}^{1}, \widetilde{\mathbf{D}}^{2}, \widetilde{\mathbf{D}}^{s}$. This is a long and very tedious project which was performed in a systematic way with the help of the basic formulae give in the Appendix. Then, an even more complicated procedure is needed to obtain the form of $\widetilde{\mathbf{R}}^{(*)} \widetilde{\mathbf{E}}^{\top}$. Finally, for the proof of the theorem we need to express the representation (24) in terms of inverse powers of $\left|\mathbf{r}-\mathbf{r}^{\prime}\right|$, where $\mathbf{r}$ is the observation point and $\mathbf{r}^{\prime}$ is the surface point of integration.

We introduce the vector

$$
\mathbf{R}=\mathbf{r}-\mathbf{r}^{\prime}=R \widehat{\mathbf{R}}
$$

and the notation

$$
\widetilde{\mathbf{E}}\left(\mathbf{r}, \mathbf{r}^{\prime}\right)=\left[\begin{array}{c|c}
\widetilde{\mathbf{E}}_{11}\left(\mathbf{r}, \mathbf{r}^{\prime}\right) & \mathbf{E}_{12}\left(\mathbf{r}, \mathbf{r}^{\prime}\right) \\
\hline \mathbf{E}_{21}\left(\mathbf{r}, \mathbf{r}^{\prime}\right) & E_{22}\left(\mathbf{r}, \mathbf{r}^{\prime}\right)
\end{array}\right]
$$

for the 4-D fundamental solution, and

$$
\widetilde{\mathbf{R}}^{(*)}\left(\partial_{\mathbf{r}^{\prime}}, \hat{\mathbf{n}}^{\prime}\right) \widetilde{\mathbf{E}}^{\top}\left(\mathbf{r}, \mathbf{r}^{\prime}\right)=\left[\begin{array}{l|l}
\widetilde{\mathbf{E}}_{11}^{t}\left(\mathbf{r}, \mathbf{r}^{\prime}\right) & \mathbf{E}_{12}^{t}\left(\mathbf{r}, \mathbf{r}^{\prime}\right) \\
\hline \mathbf{E}_{21}^{t}\left(\mathbf{r}, \mathbf{r}^{\prime}\right) & E_{22}^{t}\left(\mathbf{r}, \mathbf{r}^{\prime}\right)
\end{array}\right]
$$


for the 4-D fundamental stresses. The exact forms of (37) and (38) are given in the Appendix.

Furthermore, we define the following 3-D dyadics associated with $\widetilde{\mathbf{E}}$ :

$$
\begin{aligned}
\widetilde{\mathbf{A}}_{3}^{s}\left(\mathbf{r}^{\prime}\right) & =-\frac{1}{\rho \omega^{2}}\left(\widetilde{\mathbf{I}}_{3}-3 \widehat{\mathbf{R}} \widehat{\mathbf{R}}\right), \\
\widetilde{\mathbf{A}}_{3}^{n}\left(\mathbf{r}^{\prime}\right) & =(-1)^{n} \frac{k_{n}^{2}-(1+\varepsilon) q}{k_{1}^{2}-k_{2}^{2}} \widetilde{\mathbf{A}}_{3}^{s}\left(\mathbf{r}^{\prime}\right), \quad n=1,2, \\
\widetilde{\mathbf{A}}_{2}^{s}\left(\mathbf{r}^{\prime}\right) & =-i k_{s} \widetilde{\mathbf{A}}_{3}^{s}\left(\mathbf{r}^{\prime}\right), \\
\widetilde{\mathbf{A}}_{2}^{n}\left(\mathbf{r}^{\prime}\right) & =-i k_{n} \widetilde{\mathbf{A}}_{3}^{n}\left(\mathbf{r}^{\prime}\right), \quad n=1,2, \\
\widetilde{\mathbf{A}}_{1}^{s}\left(\mathbf{r}^{\prime}\right) & =\frac{1}{\mu}\left(\widetilde{\mathbf{I}}_{3}-\widehat{\mathbf{R}} \widehat{\mathbf{R}}\right), \\
\widetilde{\mathbf{A}}_{1}^{n}\left(\mathbf{r}^{\prime}\right) & =\frac{(-1)^{n+1}}{\lambda+2 \mu} \frac{k_{n}^{2}-q}{k_{1}^{2}-k_{2}^{2}} \widehat{\mathbf{R}} \widehat{\mathbf{R}}, \quad n=1,2,
\end{aligned}
$$

and the 3-D dyadics

$$
\begin{aligned}
& \widetilde{\mathbf{B}}_{4}^{s}\left(\mathbf{r}^{\prime}\right)=-\frac{6}{k_{s}^{2}}\left[\left(\hat{\mathbf{n}}^{\prime} \cdot \widehat{\mathbf{R}}\right)\left(\widetilde{\mathbf{I}}_{3}-5 \widehat{\mathbf{R}} \widehat{\mathbf{R}}\right)+\hat{\mathbf{n}}^{\prime} \widehat{\mathbf{R}}+\widehat{\mathbf{R}} \hat{\mathbf{n}}^{\prime}\right] \\
& \widetilde{\mathbf{B}}_{4}^{n}\left(\mathbf{r}^{\prime}\right)=(-1)^{n} \frac{k_{n}^{2}-(1+\varepsilon) q}{k_{1}^{2}-k_{2}^{2}} \widetilde{\mathbf{B}}_{4}^{s}\left(\mathbf{r}^{\prime}\right), \quad n=1,2 \\
& \widetilde{\mathbf{B}}_{3}^{s}\left(\mathbf{r}^{\prime}\right)=-i k_{s} \widetilde{\mathbf{B}}_{4}^{s}\left(\mathbf{r}^{\prime}\right), \\
& \widetilde{\mathbf{B}}_{3}^{n}\left(\mathbf{r}^{\prime}\right)=-i k_{n} \widetilde{\mathbf{B}}_{4}^{n}\left(\mathbf{r}^{\prime}\right), \quad n=1,2 \\
& \widetilde{\mathbf{B}}_{2}^{s}\left(\mathbf{r}^{\prime}\right)=3\left(\hat{\mathbf{n}}^{\prime} \cdot \widehat{\mathbf{R}}\right)\left(\widetilde{\mathbf{I}}_{3}-4 \widehat{\mathbf{R}} \widehat{\mathbf{R}}\right)+3 \widehat{\mathbf{R}} \hat{\mathbf{n}}^{\prime}+2 \hat{\mathbf{n}}^{\prime} \widehat{\mathbf{R}} \\
& \widetilde{\mathbf{B}}_{2}^{n}\left(\mathbf{r}^{\prime}\right)=\frac{(-1)^{n}}{\lambda+2 \mu} \frac{k_{n}^{2}-q}{k_{1}^{2}-k_{2}^{2}}\left[2 \mu\left(\hat{\mathbf{n}}^{\prime} \cdot \widehat{\mathbf{R}}\right)\left(\widetilde{\mathbf{I}}{ }_{3}-6 \widehat{\mathbf{R}} \widehat{\mathbf{R}}\right)\right. \\
& \left.\quad+2 \mu\left(\hat{\mathbf{n}}^{\prime} \widehat{\mathbf{R}}+\widehat{\mathbf{R}} \hat{\mathbf{n}}^{\prime}\right)-\lambda \hat{\mathbf{n}}^{\prime} \widehat{\mathbf{R}}\right]+\frac{(-1)^{n} \varepsilon q}{k_{1}^{2}-k_{2}^{2}} \hat{\mathbf{n}}^{\prime} \widehat{\mathbf{R}}, \quad n=1,2, \\
& \widetilde{\mathbf{B}}_{1}^{s}\left(\mathbf{r}^{\prime}\right)=-i k_{s}\left[\left(\hat{\mathbf{n}}^{\prime} \cdot \widehat{\mathbf{R}}\right)\left(\widetilde{\mathbf{I}}{ }_{3}-2 \widehat{\mathbf{R}} \widehat{\mathbf{R}}\right)+\widehat{\mathbf{R}} \hat{\mathbf{n}}^{\prime}\right] \\
& \widetilde{\mathbf{B}}_{1}^{n}\left(\mathbf{r}^{\prime}\right)=(-1)^{n} i k_{n} \frac{k_{n}^{2}-q}{k_{1}^{2}-k_{2}^{2}} \hat{\mathbf{n}}^{\prime} \cdot \frac{\lambda \widetilde{\mathbf{I}}_{3}+2 \mu \widehat{\mathbf{R}} \widehat{\mathbf{R}} \widehat{\mathbf{R}}}{\lambda+2 \mu} \\
& \quad+(-1)^{n+1} i k_{n} \frac{\varepsilon q}{k_{1}^{2}-k_{2}^{2}} \hat{\mathbf{n}}^{\prime} \widehat{\mathbf{R}}, \quad n=1,2
\end{aligned}
$$

associated with $\widetilde{\mathbf{R}}^{(*)} \widetilde{\mathbf{E}}^{\top}$.

With the help of the above 3-D dyadics, given by (39)-(52), we can express the coefficients of inverse powers of the distance function $\left|\mathbf{r}-\mathbf{r}^{\prime}\right|$ in the expressions $\widetilde{\mathbf{E}}$ and $\widetilde{\mathbf{R}}^{(*)} \widetilde{\mathbf{E}}^{\top}$. This step also demands long calculations which we do not include here. The end result of these calculations is the expression

$$
\begin{aligned}
\mathbf{U}(\mathbf{r})=\frac{a^{2}}{4 \pi} \sum_{n=1}^{4} & \int_{S^{2}}\left[\mathbf{H}_{n}^{1}\left(a \hat{\mathbf{r}}^{\prime}\right) e^{i k_{1}\left|\mathbf{r}-a \hat{\mathbf{r}}^{\prime}\right|}\right. \\
& \left.+\mathbf{H}_{n}^{2}\left(a \hat{\mathbf{r}}^{\prime}\right) e^{i k_{2}\left|\mathbf{r}-a \hat{\mathbf{r}}^{\prime}\right|}+\mathbf{H}_{n}^{s}\left(a \hat{\mathbf{r}}^{\prime}\right) e^{i k_{s}\left|\mathbf{r}-a \hat{\mathbf{r}}^{\prime}\right|}\right]\left|\mathbf{r}-a \hat{\mathbf{r}}^{\prime}\right|^{-n} d s\left(\hat{\mathbf{r}}^{\prime}\right)
\end{aligned}
$$


where $a$ is the radius of the sphere circumscribing $S, S^{2}$ denotes the unit sphere in $\mathbb{R}^{3}$, and the functions $\mathbf{H}^{1}, \mathbf{H}^{2}$, and $\mathbf{H}^{s}$ involve the boundary values of $\boldsymbol{\Psi}$. In fact, we obtain the expressions

$$
\mathbf{H}_{4}^{\alpha}\left(\mathbf{r}^{\prime}\right)=\boldsymbol{\Psi}\left(\mathbf{r}^{\prime}\right) \cdot \tilde{\mathbf{V}}_{4}^{\alpha}\left(\mathbf{r}^{\prime}\right), \quad \alpha=1,2, s
$$

and

$$
\mathbf{H}_{n}^{\alpha}\left(\mathbf{r}^{\prime}\right)=\boldsymbol{\Psi}\left(\mathbf{r}^{\prime}\right) \cdot \widetilde{\mathbf{V}}_{n}^{\alpha}\left(\mathbf{r}^{\prime}\right)-\widetilde{\mathbf{W}}_{n}^{\alpha}\left(\mathbf{r}^{\prime}\right) \cdot \widetilde{\mathbf{R}}\left(\partial_{\mathbf{r}^{\prime}}, \hat{\mathbf{n}}^{\prime}\right) \Psi\left(\mathbf{r}^{\prime}\right)
$$

which hold for $\alpha=1,2, s$ and $n=1,2,3$. The 4-D dyadics $\widetilde{\mathbf{W}}_{n}^{\alpha}$ express the surface field $\widetilde{\mathbf{E}}$ and they are given by

$$
\begin{aligned}
\widetilde{\mathbf{W}}_{3}^{\alpha}\left(\mathbf{r}^{\prime}\right) & =\left[\begin{array}{c|c}
\widetilde{\mathbf{A}}_{3}^{\alpha}\left(\mathbf{r}^{\prime}\right) & \mathbf{0} \\
\hline \mathbf{0} & 0
\end{array}\right], \quad \alpha=1,2, s, \\
\widetilde{\mathbf{W}}_{2}^{1}\left(\mathbf{r}^{\prime}\right) & =\left[\begin{array}{c|c}
\widetilde{\mathbf{A}}_{2}^{1}\left(\mathbf{r}^{\prime}\right) & \gamma \delta \widehat{\mathbf{R}} \\
\hline-q \kappa \eta \delta \widehat{\mathbf{R}} & 0
\end{array}\right], \\
\widetilde{\mathbf{W}}_{2}^{2}\left(\mathbf{r}^{\prime}\right) & =\left[\begin{array}{c|c}
\widetilde{\mathbf{A}}_{2}^{2}\left(\mathbf{r}^{\prime}\right) & -\gamma \delta \widehat{\mathbf{R}} \\
\hline q \kappa \eta \delta \widehat{\mathbf{R}} & 0
\end{array}\right], \\
\widetilde{\mathbf{W}}_{2}^{s}\left(\mathbf{r}^{\prime}\right) & =\left[\begin{array}{c|c}
\widetilde{\mathbf{A}}_{2}^{s}\left(\mathbf{r}^{\prime}\right) & \mathbf{0} \\
\hline \mathbf{0} & 0
\end{array}\right], \\
\widetilde{\mathbf{W}}_{1}^{1}\left(\mathbf{r}^{\prime}\right) & =\left[\begin{array}{c|c}
\widetilde{\mathbf{A}}_{1}^{1}\left(\mathbf{r}^{\prime}\right) & -i k_{1} \gamma \delta \widehat{\mathbf{R}} \\
\hline i k_{1} \kappa q \eta \delta \widehat{\mathbf{R}} & \frac{k_{1}^{2}-k_{p}^{2}}{k_{1}^{2}-k_{2}^{2}}
\end{array}\right], \\
\widetilde{\mathbf{W}}_{1}^{2}\left(\mathbf{r}^{\prime}\right) & =\left[\begin{array}{c|c}
\widetilde{\mathbf{A}}_{1}^{2}\left(\mathbf{r}^{\prime}\right) & i k_{2} \gamma \delta \widehat{\mathbf{R}} \\
\hline-i k_{2} \kappa q \eta \delta \widehat{\mathbf{R}} & -\frac{k_{2}^{2}-k_{p}^{2}}{k_{1}^{2}-k_{2}^{2}}
\end{array}\right], \\
\widetilde{\mathbf{W}}_{1}^{s}\left(\mathbf{r}^{\prime}\right) & =\left[\begin{array}{c|c|c}
\widetilde{\mathbf{A}}_{1}^{s}\left(\mathbf{r}^{\prime}\right) & \mathbf{0} \\
\hline \mathbf{0} & 0
\end{array}\right],
\end{aligned}
$$

where

$$
\delta=\frac{1}{(\lambda+2 \mu)\left(k_{1}^{2}-k_{2}^{2}\right)} .
$$


Similarly, the 4-D dyadics $\widetilde{\mathbf{V}}_{n}^{\alpha}$ express the surface traction field $\widetilde{\mathbf{R}}^{(*)} \widetilde{\mathbf{E}}^{\top}$ and assume the following forms:

$$
\begin{aligned}
& \tilde{\mathbf{V}}_{4}^{\alpha}\left(\mathbf{r}^{\prime}\right)=\left[\begin{array}{c|c}
\widetilde{\mathbf{B}}_{4}^{\alpha}\left(\mathbf{r}^{\prime}\right) & \mathbf{0} \\
\hline \mathbf{0} & 0
\end{array}\right], \quad \alpha=1,2, s, \\
& \tilde{\mathbf{V}}_{3}^{1}\left(\mathbf{r}^{\prime}\right)=\left[\begin{array}{c|c}
\widetilde{\mathbf{B}}_{3}^{1}\left(\mathbf{r}^{\prime}\right) & 2 \kappa q \eta \mu \delta \mathbf{a}\left(\mathbf{r}^{\prime}\right) \\
\hline-\gamma \delta \mathbf{a}\left(\mathbf{r}^{\prime}\right) & 0
\end{array}\right], \\
& \widetilde{\mathbf{V}}_{3}^{2}\left(\mathbf{r}^{\prime}\right)=\left[\begin{array}{c|c}
\widetilde{\mathbf{B}}_{3}^{2}\left(\mathbf{r}^{\prime}\right) & -2 \kappa q \eta \mu \delta \mathbf{a}\left(\mathbf{r}^{\prime}\right) \\
\hline \gamma \delta \mathbf{a}\left(\mathbf{r}^{\prime}\right) & 0
\end{array}\right], \\
& \widetilde{\mathbf{V}}_{3}^{s}\left(\mathbf{r}^{\prime}\right)=\left[\begin{array}{c|c}
\widetilde{\mathbf{B}}_{3}^{s}\left(\mathbf{r}^{\prime}\right) & \mathbf{0} \\
\hline \mathbf{0} & 0
\end{array}\right], \\
& \widetilde{\mathbf{V}}_{2}^{1}\left(\mathbf{r}^{\prime}\right)=\left[\begin{array}{c|c}
\widetilde{\mathbf{B}}_{2}^{1}\left(\mathbf{r}^{\prime}\right) & -2 i k_{1} \kappa q \eta \mu \delta \mathbf{a}\left(\mathbf{r}^{\prime}\right) \\
\hline i k_{1} \gamma \delta \mathbf{a}\left(\mathbf{r}^{\prime}\right) & \frac{k_{1}^{2}-k_{p}^{2}}{k_{1}^{2}-k_{2}^{2}}\left(\hat{\mathbf{n}}^{\prime} \cdot \widehat{\mathbf{R}}\right)
\end{array}\right] \text {, } \\
& \tilde{\mathbf{V}}_{2}^{2}\left(\mathbf{r}^{\prime}\right)=\left[\begin{array}{c|c}
\widetilde{\mathbf{B}}_{2}^{2}\left(\mathbf{r}^{\prime}\right) & 2 i k_{2} \kappa q \eta \mu \delta \mathbf{a}\left(\mathbf{r}^{\prime}\right) \\
\hline-i k_{2} \gamma \delta \mathbf{a}\left(\mathbf{r}^{\prime}\right) & -\frac{k_{2}^{2}-k_{p}^{2}}{k_{1}^{2}-k_{2}^{2}}\left(\hat{\mathbf{n}}^{\prime} \cdot \widehat{\mathbf{R}}\right)
\end{array}\right], \\
& \widetilde{\mathbf{V}}_{2}^{s}\left(\mathbf{r}^{\prime}\right)=\left[\begin{array}{c|c}
\widetilde{\mathbf{B}}_{2}^{s}\left(\mathbf{r}^{\prime}\right) & \mathbf{0} \\
\hline \mathbf{0} & 0
\end{array}\right] \text {, } \\
& \widetilde{\mathbf{V}}_{1}^{1}\left(\mathbf{r}^{\prime}\right)=\left[\begin{array}{c|c}
\widetilde{\mathbf{B}}_{1}^{1}\left(\mathbf{r}^{\prime}\right) & -\kappa q \eta \mu \delta\left(2 k_{1}^{2} \mathbf{b}\left(\mathbf{r}^{\prime}\right)-k_{s}^{2} \hat{\mathbf{n}}^{\prime}\right) \\
\hline-\gamma \delta k_{1}^{2} \hat{\mathbf{n}}^{\prime} \cdot \widehat{\mathbf{R}} \widehat{\mathbf{R}} & -i k_{1} \frac{k_{1}^{2}-k_{p}^{2}}{k_{1}^{2}-k_{2}^{2}}\left(\hat{\mathbf{n}}^{\prime} \cdot \widehat{\mathbf{R}}\right)
\end{array}\right], \\
& \tilde{\mathbf{V}}_{1}^{2}\left(\mathbf{r}^{\prime}\right)=\left[\begin{array}{c|c}
\widetilde{\mathbf{B}}_{1}^{2}\left(\mathbf{r}^{\prime}\right) & \kappa q \eta \mu \delta\left(2 k_{2}^{2} \mathbf{b}\left(\mathbf{r}^{\prime}\right)-k_{s}^{2} \hat{\mathbf{n}}^{\prime}\right) \\
\hline \gamma \delta k_{2}^{2} \hat{\mathbf{n}}^{\prime} \cdot \widehat{\mathbf{R}} \widehat{\mathbf{R}} & i k_{2} \frac{k_{2}^{2}-k_{p}^{2}}{k_{1}^{2}-k_{2}^{2}}\left(\hat{\mathbf{n}}^{\prime} \cdot \widehat{\mathbf{R}}\right)
\end{array}\right], \\
& \tilde{\mathbf{V}}_{1}^{s}\left(\mathbf{r}^{\prime}\right)=\left[\begin{array}{c|c}
\widetilde{\mathbf{B}}_{1}^{s}\left(\mathbf{r}^{\prime}\right) & \mathbf{0} \\
\hline \mathbf{0} & 0
\end{array}\right]
\end{aligned}
$$

where

$$
\begin{aligned}
& \mathbf{a}\left(\mathbf{r}^{\prime}\right)=\hat{\mathbf{n}}^{\prime} \cdot\left(\widetilde{\mathbf{I}}_{3}-3 \widehat{\mathbf{R}} \widehat{\mathbf{R}}\right), \\
& \mathbf{b}\left(\mathbf{r}^{\prime}\right)=\hat{\mathbf{n}}^{\prime} \cdot\left(\widetilde{\mathbf{I}}_{3}-\widehat{\mathbf{R}} \widehat{\mathbf{R}}\right)
\end{aligned}
$$

Expression (53) is obtained from the representation (24) via deformation of the surface of integration from $S$ to the circumscribing sphere of radius $a$. This deformation is possible because of Betti's third identity [10] and the lack of singularities in the region between $S$ and $S_{2}$.

Atkinson $[1,4]$ proved that for every $r \geq r_{0}>a$ the functions

$$
\lambda_{\kappa}(r, \theta, \varphi)=\frac{e^{i k(R-r)}}{R^{\kappa}}, \quad \kappa=1,2,3,4,
$$

where $R=\left|\mathbf{r}-\mathbf{r}^{\prime}\right|$ and $\left|\mathbf{r}^{\prime}\right|=a$, are analytic functions in the variable $a / r$ and that their power series expansions converge absolutely and uniformly for $r \geq r_{0}>a$ for all directions $\hat{\mathbf{r}} \in S^{2}$. Consequently, their expansions can be differentiated term by term 
with respect to $r, \theta, \varphi$ any number of times and the resulting series are also absolutely and uniformly convergent.

Applying the above Atkinson's Lemma to the representation (53) we arrive at the following form of the Atkinson-Wilcox Theorem for thermoelastic waves.

THEOREM. Let $\mathbf{U}$ be the unified scattered thermoelastic wave that corresponds to any mathematically consistent boundary condition on the scatterer, and let $a$ be the radius of the sphere that circumscribes the scatterer. Then for any $r>a$

$$
\mathbf{U}(\mathbf{r})=\frac{e^{i k_{1} r}}{r} \sum_{n=0}^{\infty} \frac{\mathbf{F}_{n}^{1}(\hat{\mathbf{r}})}{r^{n}}+\frac{e^{i k_{2} r}}{r} \sum_{n=0}^{\infty} \frac{\mathbf{F}_{n}^{2}(\hat{\mathbf{r}})}{r^{n}}+\frac{e^{i k_{s} r}}{r} \sum_{n=0}^{\infty} \frac{\mathbf{F}_{n}^{2}(\hat{\mathbf{r}})}{r^{n}}
$$

and the series converge absolutely and uniformly on the closed domain $r \geq r_{0}>a$, $\theta \in[0, \pi], \varphi \in[0,2 \pi)$.

Note that the elastothermal and the thermoelastic waves, which are represented with the first and the second series, respectively, are dissipated at the rates $d_{1}=\operatorname{Im} k_{1}$ and $d_{2}=\operatorname{Im} k_{2}$, respectively. The last series in (77), which represents the transverse elastic wave, exhibits no dissipation.

From the asymptotic forms (26)-(28) we conclude that

$$
\begin{aligned}
& \mathbf{F}_{0}^{1}(\hat{\mathbf{r}})=-i \frac{v_{1}}{\omega} \mathbf{G}^{1}(\hat{\mathbf{r}}, \hat{\mathbf{k}}), \\
& \mathbf{F}_{0}^{2}(\hat{\mathbf{r}})=-i \frac{v_{2}}{\omega} \mathbf{G}^{2}(\hat{\mathbf{r}}, \hat{\mathbf{k}}), \\
& \mathbf{F}_{0}^{s}(\hat{\mathbf{r}})=-i \frac{c_{s}}{\omega} \mathbf{G}^{s}(\hat{\mathbf{r}}, \hat{\mathbf{k}}) .
\end{aligned}
$$

Hence the leading terms of the series in (77) are recognized as the thermoelastic scattering amplitudes.

Expansion (77) can be decomposed into the displacement and the temperature fields

$$
\begin{aligned}
& \mathbf{F}_{n}^{1}(\hat{\mathbf{r}})=\left(\mathbf{u}_{n}^{1}(\hat{\mathbf{r}}), \theta_{n}^{1}(\hat{\mathbf{r}})\right), \\
& \mathbf{F}_{n}^{2}(\hat{\mathbf{r}})=\left(\mathbf{u}_{n}^{2}(\hat{\mathbf{r}}), \theta_{n}^{2}(\hat{\mathbf{r}})\right), \\
& \mathbf{F}_{n}^{s}(\hat{\mathbf{r}})=\left(\mathbf{u}_{n}^{s}(\hat{\mathbf{r}}), 0\right),
\end{aligned}
$$

for $n=0,1,2, \ldots$ to imply the expansion

$$
\mathbf{u}(\mathbf{r})=\frac{e^{i k_{1} r}}{r} \sum_{n=0}^{\infty} \frac{\mathbf{u}_{n}^{1}(\hat{\mathbf{r}})}{r^{n}}+\frac{e^{i k_{2} r}}{r} \sum_{n=0}^{\infty} \frac{\mathbf{u}_{n}^{2}(\hat{\mathbf{r}})}{r^{n}}+\frac{e^{i k_{s} r}}{r} \sum_{n=0}^{\infty} \frac{\mathbf{u}_{n}^{s}(\hat{\mathbf{r}})}{r^{n}}
$$

for the displacement field, and

$$
\theta(\mathbf{r})=\frac{e^{i k_{1} r}}{r} \sum_{n=0}^{\infty} \frac{\theta_{n}^{1}(\hat{\mathbf{r}})}{r^{n}}+\frac{e^{i k_{2} r}}{r} \sum_{n=0}^{\infty} \frac{\theta_{n}^{2}(\hat{\mathbf{r}})}{r^{n}}
$$

for the temperature field. 
For the leading coefficients in the above series, relations (30)-(32) and (34), (35) confirm that

$$
\begin{aligned}
& \mathbf{u}_{0}^{1}(\hat{\mathbf{r}})=-i \frac{v_{1}}{\omega} g_{r}^{1}(\hat{\mathbf{r}}, \hat{\mathbf{k}}) \hat{\mathbf{r}} \\
& \mathbf{u}_{0}^{2}(\hat{\mathbf{r}})=-i \frac{v_{2}}{\omega} g_{r}^{2}(\hat{\mathbf{r}}, \hat{\mathbf{k}}) \hat{\mathbf{r}} \\
& \mathbf{u}_{0}^{s}(\hat{\mathbf{r}})=-i \frac{c_{s}}{\omega}\left(g_{\theta}^{s}(\hat{\mathbf{r}}, \hat{\mathbf{k}}) \hat{\boldsymbol{\theta}}+g_{\varphi}^{s}(\hat{\mathbf{r}}, \hat{\mathbf{k}}) \hat{\boldsymbol{\varphi}}\right), \\
& \theta_{0}^{1}(\hat{\mathbf{r}})=-i \frac{v_{1}}{\omega} \ell^{1}(\hat{\mathbf{r}}, \hat{\mathbf{k}}), \\
& \theta_{0}^{2}(\hat{\mathbf{r}})=-i \frac{v_{2}}{\omega} \ell^{2}(\hat{\mathbf{r}}, \hat{\mathbf{k}}) .
\end{aligned}
$$

So, the three vectorial coefficients $\mathbf{u}_{0}^{1}, \mathbf{u}_{0}^{2}, \mathbf{u}_{0}^{s}$ and the two scalar coefficients $\theta_{0}^{1}, \theta_{0}^{2}$ are given in terms of the six scalar amplitudes $g_{r}^{1}, g_{r}^{2}, g_{\theta}^{s}, g_{\phi}^{s}, \ell^{1}$, and $\ell^{2}$.

4. Reconstruction of the expansion. For the scalar case of Atkinson's theorem, Wilcox [14] substituted the scattered field into the equation of Helmholtz and he derived a recurrence formula for the coefficients of the expansion. We can do the same here and use the uniform convergence of (77) to apply the operator $\widetilde{\mathbf{L}}$ term by term. Unfortunately, this project does not lead to a recurrence formula for the reconstruction of the coefficients $\mathbf{F}_{n}^{1}, \mathbf{F}_{n}^{2}, \mathbf{F}_{n}^{s}, n \geq 1$, in terms of $\mathbf{F}_{0}^{1}, \mathbf{F}_{0}^{2}, \mathbf{F}_{0}^{s}$. Hence, we have to decompose the fields in their spherical components according to the following procedure.

By Lemma 7 in [4] the functions

$$
\begin{aligned}
f_{n}^{1}(r) & =\frac{e^{i k_{1} r}}{r^{n+1}}, \quad n=0,1,2, \ldots, \\
f_{n}^{2}(r) & =\frac{e^{i k_{2} r}}{r^{n+1}}, \quad n=0,1,2, \ldots, \\
f_{n}^{s}(r) & =\frac{e^{i k_{s} r}}{r^{n+1}}, \quad n=0,1,2, \ldots,
\end{aligned}
$$

are linearly independent. In the interest of using this property we substitute (84), (85) into (1) and (2) and then invoke uniform convergence to differentiate term by term. From Lemma 6 in [4] we obtain for every $n=0,1,2, \ldots$ and $\alpha=1,2, s$

$$
\begin{aligned}
{[(\mu \Delta+} & \left.\left.\rho \omega^{2}\right) \widetilde{\mathbf{I}}_{3}+(\lambda+\mu) \nabla \nabla\right] \cdot\left(f_{n}^{\alpha}(r) \mathbf{u}_{n}^{\alpha}(\hat{\mathbf{r}})\right) \\
= & {\left[-k_{\alpha}^{2} \widetilde{\Lambda}_{1}+\rho \omega^{2} \widetilde{\mathbf{I}}_{3}\right] \cdot\left(f_{n}^{\alpha}(r) \mathbf{u}_{n}^{\alpha}(\hat{\mathbf{r}})\right) } \\
& +\frac{i k_{\alpha}}{r}\left[-2(n+1) \widetilde{\Lambda}_{1}+\widetilde{\Lambda}_{2}\right] \cdot\left(f_{n}^{\alpha}(r) \mathbf{u}_{n}^{\alpha}(\hat{\mathbf{r}})\right) \\
& +\frac{1}{r^{2}}\left[(n+1)(n+2) \widetilde{\Lambda}_{1}-(n+1) \widetilde{\Lambda}_{2}+\widetilde{\Lambda}_{3}\right] \cdot\left(f_{n}^{\alpha}(r) \mathbf{u}_{n}^{\alpha}(\hat{\mathbf{r}})\right)
\end{aligned}
$$

where

$$
\begin{aligned}
& \widetilde{\Lambda}_{1}=\mu \widetilde{\mathbf{I}}_{3}+(\lambda+\mu) \hat{\mathbf{r}} \hat{\mathbf{r}}, \\
& \widetilde{\Lambda}_{2}=2 \mu \widetilde{\mathbf{I}}_{3}+(\lambda+\mu)(\mathbf{D} \hat{\mathbf{r}}+\hat{\mathbf{r}} \mathbf{D}), \\
& \widetilde{\Lambda}_{3}=\mu \widetilde{\mathbf{I}}_{3} \mathcal{B}+(\lambda+\mu)(\mathbf{D D}-\hat{\mathbf{r}} \mathbf{D})
\end{aligned}
$$


with

$$
\begin{aligned}
& \nabla=\hat{\mathbf{r}} \frac{\partial}{\partial r}+\frac{1}{r} \mathbf{D} \\
& \mathbf{D}=\hat{\boldsymbol{\theta}} \frac{\partial}{\partial \theta}+\frac{\hat{\boldsymbol{\varphi}}}{\sin \theta} \frac{\partial}{\partial \varphi}
\end{aligned}
$$

and

$$
\mathcal{B}=\mathbf{D} \cdot \mathbf{D}=\frac{1}{\sin \theta} \frac{\partial}{\partial \theta}\left(\sin \frac{\partial}{\partial \theta}\right)+\frac{1}{\sin ^{2} \theta} \frac{\partial}{\partial \varphi^{2}}
$$

is the Beltrami operator.

Furthermore, we derive for $\alpha=1,2$ and every $n=0,1,2, \ldots$, the expressions

$$
\begin{aligned}
& {[\Delta+q]\left(f_{n}^{\alpha}(r) \theta_{n}^{\alpha}(\hat{\mathbf{r}})\right)=}-\left(k_{\alpha}^{2}-q\right)\left(f_{n}^{\alpha}(r) \theta_{n}^{\alpha}(\hat{\mathbf{r}})\right) \\
&-\frac{2 i k_{\alpha} n}{r}\left(f_{n}^{\alpha}(r) \theta_{n}^{\alpha}(\hat{\mathbf{r}})\right)+\frac{1}{r^{2}}[\mathcal{B}+n(n+1)]\left(f_{n}^{\alpha}(r) \theta_{n}^{\alpha}(\hat{\mathbf{r}})\right), \\
& \nabla\left(f_{n}^{\alpha}(r) \theta_{n}^{\alpha}(\hat{\mathbf{r}})\right)= i k_{\alpha}\left(f_{n}^{\alpha}(r) \theta_{n}^{\alpha}(\hat{\mathbf{r}})\right) \hat{\mathbf{r}} \\
&+\frac{1}{r}[\mathbf{D}-(n+1) \hat{\mathbf{r}}]\left(f_{n}^{\alpha}(r) \theta_{n}^{\alpha}(\hat{\mathbf{r}})\right),
\end{aligned}
$$

and

$$
\begin{aligned}
\nabla \cdot\left(f_{n}^{\alpha}(r) \mathbf{u}_{n}^{\alpha}(\hat{\mathbf{r}})\right)= & i k_{a} \hat{\mathbf{r}} \cdot\left(f_{n}^{\alpha}(r) \mathbf{u}_{n}^{\alpha}(\hat{\mathbf{r}})\right) \\
& +\frac{1}{r}[\mathbf{D}-(n+1) \hat{\mathbf{r}}] \cdot\left(f_{n}^{\alpha}(r) \mathbf{u}_{n}^{\alpha}(\hat{\mathbf{r}})\right) .
\end{aligned}
$$

Therefore, in view of (94), (101)-(103) and the linear independence of (91)-(93), Eq. (1) is reduced to three sequences of equations. The sequence

$$
\begin{aligned}
& {\left[\rho \omega^{2} \widetilde{\mathbf{I}}_{3}-k_{1}^{2} \widetilde{\Lambda}_{1}\right] \cdot \mathbf{u}_{n}^{1}(\hat{\mathbf{r}})} \\
& \quad+i k_{1}\left[-2 n \widetilde{\Lambda}_{1}+\widetilde{\Lambda}_{2}\right] \cdot \mathbf{u}_{n-1}^{1}(\hat{\mathbf{r}}) \\
& \quad+\left[n(n-1) \widetilde{\Lambda}_{1}-(n-1) \widetilde{\Lambda}_{2}+\widetilde{\Lambda}_{3}\right] \cdot \mathbf{u}_{n-2}^{1}(\hat{\mathbf{r}}) \\
& =i k_{1} \gamma \hat{\mathbf{r}} \theta_{n}^{1}(\hat{\mathbf{r}})+\gamma[\mathbf{D}-n \hat{\mathbf{r}}] \theta_{n-1}^{1}(\hat{\mathbf{r}}), \quad n=0,1,2, \ldots,
\end{aligned}
$$

that connects the coefficients of the elastothermal part, the sequence

$$
\begin{aligned}
& {\left[\rho \omega^{2} \widetilde{\mathbf{I}}_{3}-\right.}\left.k_{2}^{2} \widetilde{\Lambda}_{1}\right] \cdot \mathbf{u}_{n}^{2}(\hat{\mathbf{r}}) \\
&+i k_{2}\left[-2 n \widetilde{\Lambda}_{1}+\widetilde{\Lambda}_{2}\right] \cdot \mathbf{u}_{n-1}^{2}(\hat{\mathbf{r}}) \\
&+\left[n(n-1) \widetilde{\Lambda}_{1}-(n-1) \widetilde{\Lambda}_{2}+\widetilde{\Lambda}_{3}\right] \cdot \mathbf{u}_{n-2}^{2}(\hat{\mathbf{r}}) \\
&=i k_{2} \gamma \hat{\mathbf{r}} \theta_{n}^{2}(\hat{\mathbf{r}})+\gamma[\mathbf{D}-n \hat{\mathbf{r}}] \theta_{n-1}^{2}(\hat{\mathbf{r}}), \quad n=0,1,2, \ldots,
\end{aligned}
$$

that connects the coefficients of the thermoelastic part, and the system

$$
\begin{aligned}
& {\left[\rho \omega^{2} \widetilde{\mathbf{I}}_{3}-k_{s}^{2} \widetilde{\Lambda}_{1}\right] \cdot \mathbf{u}_{n}^{s}(\hat{\mathbf{r}})} \\
& \quad+i k_{s}\left[-2 n \widetilde{\Lambda}_{1}+\widetilde{\Lambda}_{2}\right] \cdot \mathbf{u}_{n-1}^{s}(\hat{\mathbf{r}}) \\
& \quad+\left[n(n-1) \widetilde{\Lambda}_{1}-(n-1) \widetilde{\Lambda}_{2}+\widetilde{\Lambda}_{3}\right] \cdot \mathbf{u}_{n-2}^{s}(\hat{\mathbf{r}})=\mathbf{0}, \quad n=0,1,2, \ldots,
\end{aligned}
$$

that connects the coefficients of the purely elastic transverse wave. 
Similarly, Eq. (2) is reduced to the sequence

$$
\begin{aligned}
& i k_{1} q \kappa \eta \hat{\mathbf{r}} \cdot \mathbf{u}_{n}^{1}(\hat{\mathbf{r}}) \\
& \quad+q \kappa \eta[\widetilde{\mathbf{D}}-n \hat{\mathbf{r}}] \cdot \mathbf{u}_{n-1}^{1}(\hat{\mathbf{r}}) \\
& =\left(k_{1}^{2}-q\right) \theta_{n}^{1}(\hat{\mathbf{r}})+2 i k_{1}(n-1) \theta_{n-1}^{1}(\hat{\mathbf{r}}) \\
& \quad-[\mathcal{B}+(n-1)(n-2)] \theta_{n-2}^{1}(\hat{\mathbf{r}}), \quad n=0,1,2, \ldots,
\end{aligned}
$$

and the sequence

$$
\begin{aligned}
i k_{2} q \kappa \eta \hat{\mathbf{r}} \cdot & \mathbf{u}_{n}^{2}(\hat{\mathbf{r}}) \\
& +q \kappa \eta[\widetilde{\mathbf{D}}-n \hat{\mathbf{r}}] \cdot \mathbf{u}_{n-1}^{2}(\hat{\mathbf{r}}) \\
=( & \left.k_{2}^{2}-q\right) \theta_{n}^{2}(\hat{\mathbf{r}})+2 i k_{2}(n-1) \theta_{n-1}^{2}(\hat{\mathbf{r}}) \\
& \quad-[\mathcal{B}+(n-1)(n-2)] \theta_{n-2}^{2}(\hat{\mathbf{r}}), \quad n=0,1,2, \ldots
\end{aligned}
$$

The transverse part of $\mathbf{u}$ yields no corresponding sequence, since $\mathbf{u}^{s}$ is solenoidal and therefore (29) implies that

$$
\nabla \cdot \mathbf{u}(\mathbf{r})=\nabla \cdot \mathbf{u}^{1}(\mathbf{r})+\nabla \cdot \mathbf{u}^{2}(\mathbf{r})
$$

Relations (106), that furnish the connection between the coefficients of the transverse wave, coincide with the corresponding relations in classical elasticity [4]. Therefore, we can use Proposition 2 in [4] to express

$$
\mathbf{u}_{n}^{s}(\hat{\mathbf{r}})=u_{r n}^{s}(\hat{\mathbf{r}}) \hat{\mathbf{r}}+\mathbf{u}_{t n}^{s}(\hat{\mathbf{r}})
$$

where

$$
u_{r n}^{s}(\hat{\mathbf{r}})=\mathbf{u}_{n}^{s}(\hat{\mathbf{r}}) \cdot \hat{\mathbf{r}}
$$

are the radial components, and

$$
\mathbf{u}_{t n}^{s}(\hat{\mathbf{r}})=\mathbf{u}_{n}^{s}(\hat{\mathbf{r}}) \cdot\left(\widetilde{\mathbf{I}}_{3}-\hat{\mathbf{r}} \hat{\mathbf{r}}\right)
$$

are the tangential components of $\mathbf{u}_{n}^{s}$ for each $n \geq 1$, in terms of the leading tangential coefficient $\mathbf{u}_{0}^{s}$ as it is given by $(88)$.

In particular, we obtain

$$
\begin{gathered}
i k_{s} u_{r 1}^{s}(\hat{\mathbf{r}})=-\mathbf{D} \cdot \mathbf{u}_{0}^{s}(\hat{\mathbf{r}}) \\
2 i k_{s} \mathbf{u}_{t 1}^{s}(\hat{\mathbf{r}})=\left(\widetilde{\mathbf{I}}_{3}-\hat{\mathbf{r}} \hat{\mathbf{r}}\right) \cdot \mathcal{B} \mathbf{u}_{0}^{s}(\hat{\mathbf{r}}), \\
k_{s}^{2} u_{r n}^{s}(\hat{\mathbf{r}})=i k_{s} \mathbf{K}_{n} \cdot \mathbf{u}_{n-1}^{s}(\hat{\mathbf{r}})+\hat{\mathbf{r}} \cdot \widetilde{\mathbf{L}}_{n} \cdot \mathbf{u}_{n-2}^{s}(\hat{\mathbf{r}}), \\
\frac{2 n \mu}{\lambda+\mu} k_{s}^{2} \mathbf{u}_{t n}^{s}(\hat{\mathbf{r}})=-i k_{s}\left(\widetilde{\mathbf{I}}_{3}-\hat{\mathbf{r}} \hat{\mathbf{r}}\right) \cdot \widetilde{\mathbf{L}}_{n+1} \cdot \mathbf{u}_{n-1}^{s}(\hat{\mathbf{r}}) \\
+\mathbf{D}\left[i k_{s} \mathbf{K}_{n} \cdot \mathbf{u}_{n-1}^{s}(\hat{\mathbf{r}})+\hat{\mathbf{r}} \cdot \tilde{\mathbf{L}}_{n} \cdot \mathbf{u}_{n-2}^{s}(\hat{\mathbf{r}})\right]
\end{gathered}
$$

where

$$
\mathbf{K}_{n}=\mathbf{D}+2 \frac{\mu-n(\lambda+2 \mu)}{\lambda+\mu} \hat{\mathbf{r}}
$$


and

$$
\widetilde{\mathbf{L}}_{n}=\frac{\mu}{\lambda+\mu}[(n-1)(n-2)+\mathcal{B}] \widetilde{\mathbf{I}}_{3}+(\mathbf{D}-n \hat{\mathbf{r}})(\mathbf{D}-(n-1) \hat{\mathbf{r}})
$$

for every $n=2,3, \ldots$.

We next investigate the solvability of the systems (104), (107) and (105), (108) that carry the thermoelastic coupling. We restrict consideration to the elastothermal wave $\left(\mathbf{u}^{1}, \theta^{1}\right)$, since the thermoelastic wave $\left(\mathbf{u}^{2}, \theta^{2}\right)$ behaves in exactly the same way. All we have to do to obtain the corresponding results for $\left(\mathbf{u}^{2}, \theta^{2}\right)$ is to replace the superindex 1 by the superindex 2 and the wavenumber $k_{1}$ by $k_{2}$.

We begin with the case $n=0$, where (104) and (107) yield the system

$$
\begin{aligned}
& (\lambda+2 \mu)\left(k_{p}^{2}-k_{1}^{2}\right) \hat{\mathbf{r}} \hat{\mathbf{r}} \cdot \mathbf{u}_{0}^{1}(\hat{\mathbf{r}}) \\
& +\mu\left(k_{s}^{2}-k_{1}^{2}\right)\left(\widetilde{\mathbf{I}}_{3}-\hat{\mathbf{r}} \hat{\mathbf{r}}\right) \cdot \mathbf{u}_{0}^{1}(\hat{\mathbf{r}})=i k_{1} \gamma \hat{\mathbf{r}} \theta_{0}^{1}(\hat{\mathbf{r}}), \\
& \quad i k_{1} q \kappa \eta \hat{\mathbf{r}} \cdot \mathbf{u}_{0}^{1}(\hat{\mathbf{r}})=\left(k_{1}^{2}-q\right) \theta_{0}^{1}(\hat{\mathbf{r}}) .
\end{aligned}
$$

If we introduce the spherical decomposition

$$
\mathbf{u}_{n}^{1}(\hat{\mathbf{r}})=u_{r n}^{1}(\hat{\mathbf{r}}) \hat{\mathbf{r}}+\mathbf{u}_{t n}^{1}(\hat{\mathbf{r}})
$$

where

$$
\begin{aligned}
& u_{r n}^{1}(\hat{\mathbf{r}})=\mathbf{u}_{n}^{1}(\hat{\mathbf{r}}) \cdot \hat{\mathbf{r}} \\
& \mathbf{u}_{t n}^{1}(\hat{\mathbf{r}})=\mathbf{u}_{n}^{1}(\hat{\mathbf{r}}) \cdot\left(\widetilde{\mathbf{I}}_{3}-\hat{\mathbf{r}} \hat{\mathbf{r}}\right)
\end{aligned}
$$

for $n=0,1,2, \ldots$, then the relation

$$
\frac{(\lambda+2 \mu)\left(k_{p}^{2}-k_{1}^{2}\right)}{i k_{1} q \kappa \eta}=\frac{i k_{1} \gamma}{k_{1}^{2}-q}
$$

which is a consequence of $(7),(8)$, and (10), implies that both (119) and (120) lead to

$$
\theta_{0}^{1}(\hat{\mathbf{r}})=\frac{i k_{1} q \kappa \eta}{k_{1}^{2}-q} u_{r o}^{1}(\hat{\mathbf{r}})
$$

Formula (125) shows that the temperature scattering amplitude $\ell^{1}$ is proportional to the displacement scattering amplitude $g_{r}^{1}$. In fact,

$$
\ell^{1}(\hat{\mathbf{r}}, \hat{\mathbf{k}})=\frac{i k_{1} q \kappa \eta}{k_{1}^{2}-q} g_{r}^{1}(\hat{\mathbf{r}}, \hat{\mathbf{k}})
$$

and this relation can be confirmed from formulae (87) and (98) in [5], although it was not noticed there because of the complicated expressions that $g_{r}^{1}$ and $\ell^{1}$ assume.

Projecting Eq. (119) into the orthogonal complement of $\hat{\mathbf{r}}$ we recover the well-known result

$$
\left(\widetilde{\mathbf{I}}_{3}-\hat{\mathbf{r}} \hat{\mathbf{r}}\right) \cdot \mathbf{u}_{0}^{1}(\hat{\mathbf{r}})=\mathbf{0}
$$

which proves that the scattering amplitude of the longitudinal wave $\mathbf{u}^{1}$ is radial.

In exactly the same way we obtain

$$
\theta_{0}^{2}(\hat{\mathbf{r}})=\frac{i k_{2} q \kappa \eta}{k_{2}^{2}-q} u_{r 0}^{2}(\hat{\mathbf{r}})
$$


and

$$
\left(\widetilde{\mathbf{I}}_{3}-\hat{\mathbf{r}} \hat{\mathbf{r}}\right) \cdot \mathbf{u}_{0}^{2}(\hat{\mathbf{r}})=\mathbf{0} .
$$

For $n \geq 1$ the situation is much more complicated and we start by defining, for $n=$ $1,2, \ldots$, the following dyadics, which represent the basic radial and angular decomposition:

$$
\begin{aligned}
\widetilde{\mathbf{M}}_{1}^{i} & =(\lambda+2 \mu)\left(k_{p}^{2}-k_{i}^{2}\right) \hat{\mathbf{r}} \hat{\mathbf{r}}, \quad i=1,2, \\
\widetilde{\mathbf{N}}_{1}^{i} & =\mu\left(k_{s}^{2}-k_{i}^{2}\right)(\widetilde{\mathbf{I}}-\hat{\mathbf{r}} \hat{\mathbf{r}}), \quad i=1,2, \\
\widetilde{\mathbf{M}}_{2}(n) & =(\lambda+\mu) \hat{\mathbf{r}}(\mathbf{D}-2 \hat{\mathbf{r}})-2(n-1)(\lambda+2 \mu) \hat{\mathbf{r}} \hat{\mathbf{r}}, \\
\widetilde{\mathbf{N}}_{2}(n) & =(\lambda+\mu) \mathbf{D} \hat{\mathbf{r}}-2(n-1) \mu(\widetilde{\mathbf{I}}-\hat{\mathbf{r}} \hat{\mathbf{r}}), \\
\widetilde{\mathbf{M}}_{3}(n) & =\mu \hat{\mathbf{r}} \hat{\mathbf{r}}[\mathcal{B}+(n-1)(n-2)]-n(\lambda+\mu) \hat{\mathbf{r}}[\mathbf{D}-(n-1) \hat{\mathbf{r}}], \\
\widetilde{\mathbf{N}}_{3}(n) & =\mu(\widetilde{\mathbf{I}}-\hat{\mathbf{r}} \hat{\mathbf{r}})[\mathcal{B}+(n-1)(n-2)]+(\lambda+\mu) \mathbf{D}[\mathbf{D}-(n-1) \hat{\mathbf{r}}] .
\end{aligned}
$$

Then

$$
\begin{gathered}
\rho \omega^{2} \widetilde{\mathbf{I}}_{3}-k_{1}^{2} \widetilde{\Lambda}_{1}=\widetilde{\mathbf{M}}_{1}^{1}+\widetilde{\mathbf{N}}_{1}^{1}, \\
-2 n \widetilde{\Lambda}_{1}+\widetilde{\Lambda}_{2}=\widetilde{\mathbf{M}}_{2}(n)+\widetilde{\mathbf{N}}_{2}(n), \\
(n-1)\left(n \widetilde{\Lambda}_{1}-\widetilde{\Lambda}_{2}\right)+\widetilde{\Lambda}_{3}=\widetilde{\mathbf{M}}_{3}(n)+\widetilde{\mathbf{N}}_{3}(n) .
\end{gathered}
$$

Note that

$$
\begin{array}{r}
\mathbf{D} \cdot \mathbf{u}_{n}^{1}(\hat{\mathbf{r}})=2 u_{r n}^{1}(\hat{\mathbf{r}})+\mathbf{D} \cdot \mathbf{u}_{t n}^{1}(\hat{\mathbf{r}}), \\
\hat{\mathbf{r}} \cdot \mathcal{B} \mathbf{u}_{n}^{1}(\hat{\mathbf{r}})=(\mathcal{B}-2) u_{r n}^{1}(\hat{\mathbf{r}})+\hat{\mathbf{r}} \cdot \mathcal{B} \mathbf{u}_{t n}^{1}(\hat{\mathbf{r}})
\end{array}
$$

In view of the relations (130)-(134) the elastic equation (104) is written as

$$
\begin{aligned}
\left(\widetilde{\mathbf{M}}_{1}^{1}\right. & \left.+\widetilde{\mathbf{N}}_{1}^{1}\right) \cdot \mathbf{u}_{n}^{1}(\hat{\mathbf{r}})-i k_{1} \gamma \hat{\mathbf{r}} \theta_{n}^{1}(\hat{\mathbf{r}})=\gamma(\mathbf{D}-n \hat{\mathbf{r}}) \theta_{n-1}^{1}(\hat{\mathbf{r}}) \\
& -i k_{1}\left(\widetilde{\mathbf{M}}_{2}(n)+\widetilde{\mathbf{N}}_{2}(n)\right) \cdot \mathbf{u}_{n-1}^{1}(\hat{\mathbf{r}})-\left(\widetilde{\mathbf{M}}_{3}(n)+\widetilde{\mathbf{N}}_{3}(n)\right) \cdot \mathbf{u}_{n-2}^{1}(\hat{\mathbf{r}})
\end{aligned}
$$

and the thermal equation (107) is written as

$$
\begin{aligned}
& i k_{1} q \kappa \eta u_{r n}^{1}(\hat{\mathbf{r}})-\left(k_{1}^{2}-q\right) \theta_{n}^{1}(\hat{\mathbf{r}})=2 i k_{1}(n-1) \theta_{n-1}^{1}(\hat{\mathbf{r}}) \\
& \quad-q \kappa \eta(\mathbf{D}-n \hat{\mathbf{r}}) \cdot \mathbf{u}_{n-1}^{1}(\hat{\mathbf{r}})-[\mathcal{B}+(n-1)(n-2)] \theta_{n-2}^{1}(\hat{\mathbf{r}}) .
\end{aligned}
$$

A further decomposition of (141) into radial and angular parts yields

$$
\begin{aligned}
& \hat{\mathbf{r}} \cdot \widetilde{\mathbf{M}}_{1}^{1} \cdot \mathbf{u}_{n}^{1}(\hat{\mathbf{r}})-i k_{1} \gamma \theta_{n}^{1}(\hat{\mathbf{r}}) \\
& \quad=-n \gamma \theta_{n-1}^{1}(\hat{\mathbf{r}})-i k_{1} \hat{\mathbf{r}} \cdot \widetilde{\mathbf{M}}_{2}(n) \cdot \mathbf{u}_{n-1}^{1}(\hat{\mathbf{r}})-\hat{\mathbf{r}} \cdot \widetilde{\mathbf{M}}_{3}(n) \cdot \mathbf{u}_{n-2}^{1}(\hat{\mathbf{r}})
\end{aligned}
$$

for the radial projection and

$$
\widetilde{\mathbf{N}}_{1}^{1} \cdot \mathbf{u}_{n}^{1}(\hat{\mathbf{r}})=\gamma \mathbf{D} \theta_{n-1}^{1}(\hat{\mathbf{r}})-i k_{1} \widetilde{\mathbf{N}}_{2}(n) \cdot \mathbf{u}_{n-1}^{1}(\hat{\mathbf{r}})-\widetilde{\mathbf{N}}_{3}(n) \cdot \mathbf{u}_{n-2}^{1}(\hat{\mathbf{r}})
$$

for the tangential projection. 
Equation (144) cannot be solved with respect to $\mathbf{u}_{n}^{1}$ since $\tilde{\mathbf{N}}_{1}^{1}$ is a genuine projection from $\mathbb{R}^{3}$ and $\mathbb{R}^{2}$ and therefore it is not invertible. Nevertheless, if the spherical decomposition (121) is considered, then the projection operator $\widetilde{\mathbf{I}}_{3}-\hat{\mathbf{r}} \hat{\mathbf{r}}$ defining $\widetilde{\mathbf{N}}_{1}^{1}$ degenerates to the identity on the orthogonal complement $\{\hat{\mathbf{r}}\}^{\perp}$ and therefore it is invertible in $\{\hat{\mathbf{r}}\}^{\perp}$.

In this case (144) implies

$$
\mu\left(k_{s}^{2}-k_{1}^{2}\right) \mathbf{u}_{t n}^{1}(\hat{\mathbf{r}})=\gamma \mathbf{D} \theta_{n-1}^{1}(\hat{\mathbf{r}})-i k_{1} \tilde{\mathbf{N}}_{2}(n) \cdot \mathbf{u}_{n-1}^{1}(\hat{\mathbf{r}})-\widetilde{\mathbf{N}}_{3}(n) \cdot \mathbf{u}_{n-2}^{1}(\hat{\mathbf{r}})
$$

and since $k_{s}^{2} \neq k_{1}^{2}$, Eq. (145) furnishes the tangential component of $\mathbf{u}_{n}^{1}$, for every $n=$ $1,2, \ldots$

Next we use (124) to rewrite (143) as

$$
\begin{aligned}
& i k_{1} q \kappa \eta u_{r n}^{1}(\hat{\mathbf{r}})-\left(k_{1}^{2}-q\right) \theta_{n}^{1}(\hat{\mathbf{r}})=\frac{i n\left(k_{1}^{2}-q\right)}{k_{1}} \theta_{n-1}^{1}(\hat{\mathbf{r}}) \\
& \quad-\frac{k_{1}^{2}-q}{\gamma} \hat{\mathbf{r}} \cdot \widetilde{\mathbf{M}}_{2}(n) \cdot \mathbf{u}_{n-1}^{1}(\hat{\mathbf{r}})+\frac{i\left(k_{1}^{2}-q\right)}{k_{1} \gamma} \hat{\mathbf{r}} \cdot \widetilde{\mathbf{M}}_{3}(n) \cdot \mathbf{u}_{n-2}^{1}(\hat{\mathbf{r}}) .
\end{aligned}
$$

The radial component $u_{r n}^{1}$ and $\theta_{n}^{1}$ are coupled by the system of equations (142) and (146). This system, as it stands, is inconsistent since the left-hand sides coincide but the right-hand sides do not. Nevertheless, the two equations come from the two governing equations of the Biot system (1), (2). Hence, (142) and (146) have to be consistent and this demands that their right-hand sides coincide.

Consequently, the equation

$$
\begin{aligned}
& \frac{i n\left(k_{1}^{2}-q\right)}{k_{1}} \theta_{n-1}^{1}(\hat{\mathbf{r}})-\frac{k_{1}^{2}-q}{\gamma} \hat{\mathbf{r}} \cdot \widetilde{\mathbf{M}}_{2}(n) \cdot \mathbf{u}_{n-1}^{1}(\hat{\mathbf{r}}) \\
& \quad+\frac{i\left(k_{1}^{2}-q\right)}{k_{1} \gamma} \hat{\mathbf{r}} \cdot \widetilde{\mathbf{M}}_{3}(n) \cdot \mathbf{u}_{n-2}^{1}(\hat{\mathbf{r}}) \\
&=2 i k_{1}(n-1) \theta_{n-1}^{1}(\hat{\mathbf{r}})-q \kappa \eta(\mathbf{D}-n \hat{\mathbf{r}}) \cdot \mathbf{u}_{n-1}^{1}(\hat{\mathbf{r}}) \\
&-[\mathcal{B}+(n-1)(n-2)] \theta_{n-2}^{1}(\hat{\mathbf{r}}),
\end{aligned}
$$

which is the condition of consistency for the system (142), (146), provides a second independent equation connecting $u_{r(n-1)}^{1}$ and $\theta_{n-1}^{1}$.

Indeed, if we use (139), (140) and the expression

$$
\begin{aligned}
\mathbf{D} \cdot \mathbf{u}_{t n}^{1}(\hat{\mathbf{r}})= & \frac{\gamma}{\mu\left(k_{s}^{2}-k_{1}^{2}\right)} \mathcal{B} \theta_{n-1}^{1}(\hat{\mathbf{r}}) \\
& -\frac{i k_{1}}{\mu\left(k_{s}^{2}-k_{1}^{2}\right)} \mathbf{D} \cdot\left(\widetilde{\mathbf{N}}_{2}(n) \cdot \mathbf{u}_{n-1}^{1}(\hat{\mathbf{r}})\right) \\
& -\frac{1}{\mu\left(k_{s}^{2}-k_{1}^{2}\right)} \mathbf{D} \cdot\left(\tilde{\mathbf{N}}_{3}(n) \cdot \mathbf{u}_{n-2}^{1}(\hat{\mathbf{r}})\right)
\end{aligned}
$$


for $n=1,2, \ldots$, then Eq. (147), for $n+1$ in place of $n$, yields

$$
\begin{aligned}
& i k_{1}(\lambda+2 \mu)\left[(n+1) \varepsilon q-2 n\left(k_{2}^{2}-k_{p}^{2}\right)\right] u_{r n}^{1}(\hat{\mathbf{r}}) \\
& +\gamma\left[(n-1) k_{1}^{2}+(n+1) q\right] \theta_{n}^{1}(\hat{\mathbf{r}}) \\
& =i k_{1}\left[1-\frac{\left.(\lambda+2 \mu) k_{2}^{2}-\mu q\right]}{\mu\left(k_{s}^{2}-k_{1}^{2}\right)}\right]\left[\gamma \mathcal{B} \theta_{n-1}^{1}(\hat{\mathbf{r}})\right. \\
& \left.\quad-i k_{1} \mathbf{D} \cdot\left(\widetilde{\mathbf{N}}_{2}(n) \cdot \mathbf{u}_{n-1}^{1}(\hat{\mathbf{r}})\right)-\mathbf{D} \cdot\left(\widehat{\mathbf{N}}_{3}(n) \cdot \mathbf{u}_{n-2}^{1}(\hat{\mathbf{r}})\right)\right] \\
& +\left(k_{1}^{2}-q\right) \hat{\mathbf{r}} \cdot \widetilde{\mathbf{M}}_{3}(n+1) \cdot \mathbf{u}_{n-1}^{1}(\hat{\mathbf{r}}) \\
& -i k_{1} \gamma[\mathcal{B}+n(n-1)] \theta_{n-1}^{1}(\hat{\mathbf{r}}) .
\end{aligned}
$$

Equations (142) and (149) form now a new system for the determination of $u_{r n}^{1}$ and $\theta_{n}^{1}$. The determinant of the coefficients of the unknowns is given by

$$
D(n)=2 i n k_{1}(\lambda+2 \mu)\left(k_{1}^{2}-k_{2}^{2}\right)\left(k_{1}^{2}-q\right) .
$$

Since $k_{1}^{2} \neq k_{2}^{2}$ and $k_{1}^{2} \neq q$ it follows that all these new systems are uniquely solvable for every $n=1,2, \ldots$ and they express $u_{r n}^{1}$ and $\theta_{n}^{1}$ in terms of previous known coefficients.

More precisely we obtain

$$
\begin{aligned}
u_{r n}^{1}(\hat{\mathbf{r}}) & =\frac{D^{u}(n)}{D(n)}, \quad n=1,2, \ldots, \\
\theta_{n}^{1}(\hat{\mathbf{r}})=\frac{D^{\theta}(n)}{D(n)}, & n=1,2, \ldots,
\end{aligned}
$$

where

$$
\begin{aligned}
D^{u}(n)=\gamma[ & \left.(n-1) k_{1}^{2}+(n+1) q\right]\left\{2 i k_{1}(n-1) \theta_{n-1}^{1}(\hat{\mathbf{r}})\right. \\
& \left.-q \kappa \eta(\mathbf{D}-n \hat{\mathbf{r}}) \cdot \mathbf{u}_{n-1}^{1}(\hat{\mathbf{r}})-[\mathcal{B}+(n-1)(n-2)] \theta_{n-2}^{1}(\hat{\mathbf{r}})\right\} \\
& +i k_{1}\left(k_{1}^{2}-q\right)\left[1-\frac{\left.(\lambda+2 \mu) k_{2}^{2}-\mu q\right]}{\mu\left(k_{s}^{2}-k_{1}^{2}\right)}\right]\left[\gamma \mathcal{B} \theta_{n-1}^{1}(\hat{\mathbf{r}})\right. \\
& \left.-i k_{1} \mathbf{D} \cdot\left(\widetilde{\mathbf{N}}_{2}(n) \cdot \mathbf{u}_{n-1}^{1}(\hat{\mathbf{r}})\right)-\mathbf{D} \cdot\left(\widetilde{\mathbf{N}}_{3}(n) \cdot \mathbf{u}_{n-2}^{1}(\hat{\mathbf{r}})\right)\right] \\
& +\left(k_{1}^{2}-q\right)^{2} \hat{\mathbf{r}} \cdot \widetilde{\mathbf{M}}_{3}(n+1) \cdot \mathbf{u}_{n-1}^{1}(\hat{\mathbf{r}}) \\
& -i k_{1} \gamma\left(k_{1}^{2}-q\right)[\mathcal{B}+n(n-1)] \theta_{n-1}^{1}(\hat{\mathbf{r}}), \\
D^{\theta}(n)=- & k_{1}^{2} q \kappa \eta\left[1-\frac{(\lambda+2 \mu) k_{2}^{2}-\mu q}{\mu\left(k_{s}^{2}-k_{1}^{2}\right)}\right]\left[\gamma \mathcal{B} \theta_{n-1}^{1}(\hat{\mathbf{r}})\right. \\
& \left.\quad-i k_{1} \mathbf{D} \cdot\left(\widetilde{\mathbf{N}}_{2}(n) \cdot \mathbf{u}_{n-1}^{1}(\hat{\mathbf{r}})\right)-\mathbf{D} \cdot\left(\widetilde{\mathbf{N}}_{3}(n) \cdot \mathbf{u}_{n-2}^{1}(\hat{\mathbf{r}})\right)\right] \\
+ & i k_{1}\left(k_{1}^{2}-q\right) q \kappa \eta \hat{\mathbf{r}} \cdot \widetilde{\mathbf{M}}_{3}(n+1) \cdot \mathbf{u}_{n-1}^{1}(\hat{\mathbf{r}}) \\
+ & k_{1}^{2} q \kappa \eta \gamma[\mathcal{B}+n(n-1)] \theta_{n-1}^{1}(\hat{\mathbf{r}}) \\
& -i k_{1}(\lambda+2 \mu)\left[(n+1) \varepsilon q-2 n\left(k_{2}^{2}-k_{p}^{2}\right)\right]\left\{2 i k_{1}(n-1) \theta_{n-1}^{1}(\hat{\mathbf{r}})\right. \\
& \left.\quad-q \kappa \eta(\mathbf{D}-n \hat{\mathbf{r}}) \cdot \mathbf{u}_{n-1}^{1}(\hat{\mathbf{r}})-[\mathcal{B}+(n-1)(n-2)] \theta_{n-2}^{1}(\hat{\mathbf{r}})\right\}
\end{aligned}
$$

and $D(n)$ is given by $(150)$. 
Recapitulating our results we see that all five series in (84), (85) can be completely reconstructed once the leading coefficients of the three elastic series in (84) are given. The algorithm of reconstruction demands the following steps.

Step 1: Obtain $\theta_{0}^{1}$ in terms of $u_{r 0}^{1}$ from (125).

Step 2: Repeat Step 1 for $\theta_{0}^{2}$.

Step 3: For $n=1,2, \ldots$ obtain $u_{r n}^{1}$ and $\theta_{n}^{1}$ from (151), (152).

Step 4: Repeat Step 3 for $u_{r n}^{2}$ and $\theta_{n}^{2}$.

Step 5: For $n=1,2, \ldots$ obtain $\mathbf{u}_{t n}^{1}$ from (145).

Step 6: Repeat Step 5 for $\mathbf{u}_{t n}^{2}$.

Step 7: For $n=1,2, \ldots$ obtain $u_{r n}^{s}$ and $\mathbf{u}_{t n}^{s}$ in terms of $\mathbf{u}_{0}^{s}$, from (113)-(116).

Therefore, the radial scattering amplitude $g_{r}^{1}$ reconstructs the first series on the righthand side of (84) and (85). Similarly, the radial scattering amplitude $g_{r}^{2}$ reconstructs the second series on the right-hand side of (84) and (85). Finally, the third series on the right-hand side of (84) is reconstructed from the angular scattering amplitudes $g_{\theta}^{s}$ and $g_{\phi}^{s}$. All together, the eleven scalar series appearing in (84) and (85) are completely reconstructable from four scalar functions, the amplitudes $g_{r}^{1}, g_{\mathbf{r}}^{2}, g_{\theta}^{s}$, and $g_{\phi}^{s}$.

5. Reduction to acoustics, electromagnetics, and elasticity. In this section, we will show how all previous versions $[1,4,14,15]$ of the Atkinson-Wilcox theorem can be recovered by considering special limits of the parameters.

We start with classical elasticity [4]. This is obtained by considering the limiting case where the coupling constants $\varepsilon, \gamma, \eta$ tend to zero. Then we obtain

$$
\begin{aligned}
& \lim _{\varepsilon \rightarrow 0+} k_{1}^{2}=k_{p}^{2}, \\
& \lim _{\varepsilon \rightarrow 0+} k_{2}^{2}=q
\end{aligned}
$$

and by virtue of

$$
\begin{gathered}
\mu\left(k_{s}^{2}-k_{p}^{2}\right)=(\lambda+\mu) k_{p}^{2}, \\
\lim _{\varepsilon \rightarrow 0+}\left[1-\frac{(\lambda+2 \mu) k_{2}^{2}-\mu q}{\mu\left(k_{s}^{2}-k_{1}^{2}\right)}\right]=\frac{k_{p}^{2}-q}{k_{p}^{2}} .
\end{gathered}
$$

The determinant (150) assumes the limiting value

$$
\lim _{\varepsilon \rightarrow 0+} D(n)=2 n i k_{p}(\lambda+2 \mu)\left(k_{p}^{2}-q\right)^{2}
$$

and the radial component $u_{r n}^{1}$, given by (151), (153), (150), reduces to

$$
\begin{aligned}
\lim _{\varepsilon \rightarrow 0+} u_{r n}^{1}(\hat{\mathbf{r}})= & \frac{1}{2 n i k_{p}(\lambda+2 \mu)} \mathbf{D} \cdot \widetilde{\mathbf{N}}_{2}(n) \cdot \mathbf{u}_{n-1}^{p}(\hat{\mathbf{r}}) \\
& +\frac{1}{2 n i k_{p}(\lambda+2 \mu)} \hat{\mathbf{r}} \cdot \widetilde{\mathbf{M}}_{3}(n+1) \cdot \mathbf{u}_{n-1}^{p}(\hat{\mathbf{r}}) \\
& \quad-\frac{1}{2 n k_{p}^{2}(\lambda+2 \mu)} \mathbf{D} \cdot \widetilde{\mathbf{N}}_{3}(n) \cdot \mathbf{u}_{n-2}^{p}(\hat{\mathbf{r}}) \\
= & u_{r n}^{p}(\hat{\mathbf{r}})
\end{aligned}
$$


Substituting (133), (134), and (135) into (160) and using relations (99) and (100) we conclude that

$$
\begin{aligned}
u_{r n}^{p}(\hat{\mathbf{r}})=\frac{1}{2 n i k_{p}(\lambda+2 \mu)}\left\{(\lambda+\mu) \mathcal{B} u_{r(n-1)}^{p}(\hat{\mathbf{r}})+\mu \hat{\mathbf{r}} \cdot[\mathcal{B}+n(n-1)] \mathbf{u}_{n-1}^{p}(\hat{\mathbf{r}})\right. \\
-[2(n-1) \mu+(n+1)(\lambda+\mu)] \mathbf{D} \cdot \mathbf{u}_{n-1}^{p}(\hat{\mathbf{r}}) \\
\left.+[4(n-1) \mu+n(n+1)(\lambda+\mu)] u_{r(n-1)}^{p}(\hat{\mathbf{r}})\right\} \\
-\frac{1}{2 n k_{p}^{2}(\lambda+2 \mu)}\left\{\mu \mathbf{D} \cdot[\mathcal{B}+(n-1)(n-2)] \mathbf{u}_{n-2}^{p}(\hat{\mathbf{r}})\right. \\
-2 \mu \hat{\mathbf{r}} \cdot[\mathcal{B}+(n-1)(n-2)] \mathbf{u}_{n-2}^{p}(\hat{\mathbf{r}}) \\
\left.+(\lambda+\mu) \mathcal{B}[\mathbf{D}-(n-1) \hat{\mathbf{r}}] \cdot \mathbf{u}_{n-2}^{p}(\hat{\mathbf{r}})\right\}
\end{aligned}
$$

where we have also used the fact that if a vector field is radial then it belongs to the kernel of the operator $\mathbf{D}-2 \hat{\mathbf{r}}$. That is,

$$
(\mathbf{D}-2 \hat{\mathbf{r}}) \cdot(\hat{\mathbf{r}} f(\hat{\mathbf{r}}))=0
$$

or

$$
(\mathbf{D} \cdot \hat{\mathbf{r}}) f(\hat{\mathbf{r}})=2 f(\hat{\mathbf{r}})
$$

which shows that 2 is an eigenvalue of the scalar operator $\mathbf{D} \cdot \hat{\mathbf{r}}$. Further use of (163) allows us to rewrite (161) as

$$
\begin{gathered}
u_{r n}^{p}(\hat{\mathbf{r}})=\frac{1}{2 n i k_{p}(\lambda+2 \mu)}\left\{(\lambda+\mu) \mathcal{B} u_{r(n-1)}^{p}(\hat{\mathbf{r}})+\mu \hat{\mathbf{r}} \cdot[\mathcal{B}+n(n-1)] \mathbf{u}_{n-1}^{p}(\hat{\mathbf{r}})\right. \\
-2(n-1) \mu(\mathbf{D}-2 \hat{\mathbf{r}}) \cdot \mathbf{u}_{n-1}^{p}(\hat{\mathbf{r}}) \\
\left.\quad-(n+1)(\lambda+\mu)(\mathbf{D}-n \hat{\mathbf{r}}) \cdot \mathbf{u}_{n-1}^{p}(\hat{\mathbf{r}})\right\} \\
-\frac{\lambda+\mu}{2 n k_{p}^{2}(\lambda+2 \mu)}(\mathbf{D}-2 \hat{\mathbf{r}}) \cdot \widetilde{\mathbf{L}}_{n} \cdot \mathbf{u}_{n-2}^{p}(\hat{\mathbf{r}})
\end{gathered}
$$

where $\widetilde{\mathbf{L}}_{n}$ is given by (118).

Using (118) for $(n+1)$ once more we arrive at

$$
\begin{aligned}
u_{r n}^{p}(\hat{\mathbf{r}})= & \frac{\lambda+\mu}{2 n k_{p}^{2}(\lambda+2 \mu)}(\mathbf{D}-2 \hat{\mathbf{r}}) \cdot\left[2 i k_{p}(n-1) \frac{\mu}{\lambda+\mu} \mathbf{u}_{n-1}^{p}(\hat{\mathbf{r}})-\widetilde{\mathbf{L}}_{n} \cdot \mathbf{u}_{n-2}^{p}(\hat{\mathbf{r}})\right] \\
& -\frac{i k_{p}(\lambda+\mu)}{2 n k_{p}^{2}(\lambda+2 \mu)}\left[\mathcal{B} u_{r(n-1)}^{p}(\hat{\mathbf{r}})+\hat{\mathbf{r}} \cdot \widetilde{\mathbf{L}}_{n+1} \cdot \mathbf{u}_{n-1}^{p}(\hat{\mathbf{r}})\right],
\end{aligned}
$$

which coincides with formula (58) in [4] that provides the radial component of $\mathbf{u}_{n}^{p}$ in classical elasticity.

The tangential component of $\mathbf{u}_{n}^{1}$, as it is given by (145), in the limit as $\varepsilon$ tends to zero furnishes the following tangential component of classical elasticity:

$$
\begin{aligned}
\mathbf{u}_{t n}^{p}(\hat{\mathbf{r}}) & =\lim _{\varepsilon \rightarrow 0+} \mathbf{u}_{t n}^{1}(\hat{\mathbf{r}}) \\
& =-\frac{i k_{p}}{(\lambda+\mu) k_{p}^{2}} \tilde{\mathbf{N}}_{2}(n) \cdot \mathbf{u}_{n-1}^{p}(\hat{\mathbf{r}})-\frac{1}{(\lambda+\mu) k_{p}^{2}} \tilde{\mathbf{N}}_{3}(n) \cdot \mathbf{u}_{n-2}^{p}(\hat{\mathbf{r}}) .
\end{aligned}
$$


Using (133), (135) and some calculations we can rewrite (166) as

$$
\begin{aligned}
\mathbf{u}_{t n}^{p}(\hat{\mathbf{r}})=\frac{2 i k_{p}(n-1) \mu}{(\lambda+\mu) k_{p}^{2}} \mathbf{u}_{t(n-1)}^{p}(\hat{\mathbf{r}}) & +\frac{1}{i k_{p}} \mathbf{D} u_{r(n-1)}^{p}(\hat{\mathbf{r}}) \\
-\frac{1}{k_{p}^{2}}(\widetilde{\mathbf{I}}-\hat{\mathbf{r}} \hat{\mathbf{r}}) \cdot\left\{\frac{\mu}{\lambda+\mu}[\mathcal{B}+(n-1)(n-2)] \widetilde{\mathbf{I}}_{3}\right. & +(\mathbf{D}-\mathbf{n} \hat{\mathbf{r}})(\mathbf{D}-(n-1) \hat{\mathbf{r}})\} \cdot \mathbf{u}_{n-2}^{p}(\hat{\mathbf{r}}),
\end{aligned}
$$

which coincides with relation (60) in [4] that provides the corresponding tangential component in classical elasticity. Finally, since the relative formulae for the transverse wave in thermoelasticity are exactly the same with those in classical elasticity, it follows that the Atkinson-Wilcox theorem in elasticity is completely recovered from its thermoelastic version.

Then we move to the next in lower complication case which is that of electromagnetism. The electromagnetic waves correspond to the transverse part of the solution of Eq. (1) in the decoupled case $\gamma=0$, when we choose $\lambda=-\mu$. In this case, formulae (113)-(118) derive, for $n=1,2,3, \ldots$,

$$
\begin{aligned}
& E_{r 1}(\hat{\mathbf{r}})=-\frac{1}{i k} \mathbf{D} \cdot \mathbf{E}_{0}(\hat{\mathbf{r}}), \\
& E_{r n}(\hat{\mathbf{r}})=\frac{1}{2 i k n} \hat{\mathbf{r}} \cdot[\mathcal{B}+n(n-1)] \mathbf{E}_{n-1}(\hat{\mathbf{r}})
\end{aligned}
$$

for the radial components of the coefficients of the electric field, and

$$
\mathbf{E}_{t n}(\hat{\mathbf{r}})=\frac{1}{2 i k_{n}}\left(\widetilde{\mathbf{I}}_{3}-\hat{\mathbf{r}} \hat{\mathbf{r}}\right) \cdot[\mathcal{B}+n(n-1)] \mathbf{E}_{n-1}(\hat{\mathbf{r}})
$$

for the corresponding tangential components, where $k=k_{s}$. Expressions (168)-(170) coincide with the corresponding expressions in [15] that were given by Wilcox for electromagnetic scattering.

Finally, the original acoustic version of the theorem [1], and the recurrence formulae given in [14], can be recovered from the longitudinal part of the decoupled case $\gamma=0$, when $\lambda=-\mu$ and $\mathbf{u}=u \hat{\mathbf{k}}$. That will conclude the relations

$$
u_{n}(\hat{\mathbf{r}})=\frac{1}{2 i k_{n}}[\mathcal{B}+n(n-1)] u_{n-1}(\hat{\mathbf{r}})
$$

for every $n=1,2, \ldots$ which were obtained by Wilcox in [14].

Concluding, we see that all previous versions of the Atkinson-Wilcox theorem are recoverable from the general thermoelastic scattering problem. It is of interest to investigate what general conditions on the form of a hyperbolic operator, or on the reduced spectral elliptic operator after we consider harmonic dependence, will allow for an Atkinson-Wilcox type expansion theorem for the general scattered field.

Appendix: Basic formulae. Let

$$
\mathbf{R}=\mathbf{r}-\mathbf{r}^{\prime}=R \widehat{\mathbf{R}}
$$


where $\widehat{\mathbf{R}}$ denotes the unit vector in the direction of $\mathbf{R}$. We define the generic function

$$
f(R)=\frac{e^{i k R}}{R}
$$

which provides the following fundamental solutions for the Helmholtz equation:

$$
\begin{aligned}
& f_{1}(R)=\frac{e^{i k_{1} R}}{R}, \\
& f_{2}(R)=\frac{e^{i k_{2} R}}{R}, \\
& f_{s}(R)=\frac{e^{i k_{s} R}}{R}, \\
& f_{p}(R)=\frac{e^{i k_{p} R}}{R} .
\end{aligned}
$$

Straightforward calculations derive the vector field

$$
\nabla_{\mathbf{r}} f(R)=f^{\prime}(R) \widehat{\mathbf{R}},
$$

the dyadic field

$$
\nabla_{\mathbf{r}} \nabla_{\mathbf{r}} f(R)=f^{\prime \prime}(R) \widehat{\mathbf{R}} \widehat{\mathbf{R}}+\frac{f^{\prime}(R)}{R}\left(\widetilde{\mathbf{I}}_{3}-\widehat{\mathbf{R}} \widehat{\mathbf{R}}\right),
$$

and the triadic field

$$
\begin{aligned}
& \nabla_{\mathbf{r}} \nabla_{\mathbf{r}} \nabla_{\mathbf{r}} f(R)=f^{\prime \prime \prime}(R) \widehat{\mathbf{R}} \widehat{\mathbf{R}} \widehat{\mathbf{R}}+\left(\frac{f^{\prime \prime}(R)}{R}-\frac{f^{\prime}(R)}{R^{2}}\right) {\left[\widehat{\mathbf{R}}\left(\widetilde{\mathbf{I}}_{3}-\widehat{\mathbf{R}} \widehat{\mathbf{R}}\right)\right.} \\
&\left.+\widehat{\mathbf{R}}\left(\widetilde{\mathbf{I}}_{3}-\widehat{\mathbf{R}} \widehat{\mathbf{R}}\right)^{213}+\left(\widetilde{\mathbf{I}}_{3}-\widehat{\mathbf{R}} \widehat{\mathbf{R}}\right) \widehat{\mathbf{R}}\right]
\end{aligned}
$$

where the exponent 213 specifies the order of the tensorial factors in the corresponding triadic, i.e.,

$$
\operatorname{abc}^{213}=\text { bac. }
$$

Any gradient $\nabla_{\mathbf{r}}$ can be replaced by the gradient $\nabla_{\mathbf{r}^{\prime}}$ in (A.6), (A.7), (A.8) with a simultaneous change of sign, i.e.,

$$
\nabla_{\mathbf{r}}=-\nabla_{\mathbf{r}^{\prime}}=\nabla_{\mathbf{R}}
$$

The components of the above tensorial fields are obtained from

$$
\begin{aligned}
f^{\prime}(R) & =\left(i k-\frac{1}{R}\right) f(R), \\
f^{\prime \prime}(R) & =\left(-k^{2}-\frac{2 i k}{R}+\frac{2}{R^{2}}\right) f(R), \\
f^{\prime \prime \prime}(R) & =\left(-i k^{3}+\frac{3 k^{2}}{R}+\frac{6 i k}{R^{2}}-\frac{6}{R^{3}}\right) f(R) .
\end{aligned}
$$


Using appropriate scalar and vector invariants of (A.7) and (A.8) we evaluate the surface tractions that are generated by the dyadic fields $\nabla \nabla f$ and $f \widetilde{\mathbf{I}}_{3}$. These are

$$
\begin{aligned}
\widetilde{\mathbf{T}}\left(\partial_{\mathbf{r}^{\prime}}, \hat{\mathbf{n}}^{\prime}\right)\left(\nabla_{\mathbf{r}} \nabla_{\mathbf{r}} f(R)\right)=- & f^{\prime \prime \prime}(R) \hat{\mathbf{n}}^{\prime} \cdot\left(\lambda \widetilde{\mathbf{I}}_{3}+2 \mu \widehat{\mathbf{R}} \widehat{\mathbf{R}}\right) \widehat{\mathbf{R}} \\
& -2\left(\frac{f^{\prime \prime}(R)}{R}-\frac{f^{\prime}(R)}{R^{2}}\right) \\
& {\left[\mu\left(\hat{\mathbf{n}}^{\prime} \cdot \widehat{\mathbf{R}}\right)\left(\widetilde{\mathbf{I}}_{3}-3 \widehat{\mathbf{R}} \widehat{\mathbf{R}}\right)\right.} \\
& \left.+\mu \widehat{\mathbf{R}} \hat{\mathbf{n}}^{\prime}+(\lambda+\mu) \hat{\mathbf{n}}^{\prime} \widehat{\mathbf{R}}\right]
\end{aligned}
$$

and

$$
\widetilde{\mathbf{T}}\left(\partial_{\mathbf{r}^{\prime}}, \hat{\mathbf{n}}^{\prime}\right)\left(f(R) \widetilde{\mathbf{I}}_{3}\right)=-f^{\prime}(R)\left[\mu\left(\hat{\mathbf{n}}^{\prime} \cdot \widehat{\mathbf{R}}\right) \widetilde{\mathbf{I}}_{3}+\mu \widehat{\mathbf{R}} \hat{\mathbf{n}}^{\prime}+\lambda \hat{\mathbf{n}}^{\prime} \widehat{\mathbf{R}}\right] .
$$

With the help of the above formulae we derive the following analytic expressions for the fundamental dyadic (37):

$$
\begin{aligned}
\widetilde{\mathbf{E}}_{11}\left(\mathbf{r}, \mathbf{r}^{\prime}\right)= & -\frac{\left(k_{p}^{2}-k_{2}^{2}\right) f_{1}^{\prime \prime}(R)-\left(k_{p}^{2}-k_{1}^{2}\right) f_{2}^{\prime \prime}(R)-\left(k_{1}^{2}-k_{2}^{2}\right) f_{s}^{\prime \prime}(R)}{\rho \omega^{2}\left(k_{1}^{2}-k_{2}^{2}\right)} \widehat{\mathbf{R}} \widehat{\mathbf{R}} \\
& -\frac{\left(k_{p}^{2}-k_{2}^{2}\right) f_{1}^{\prime}(R)-\left(k_{p}^{2}-k_{1}^{2}\right) f_{2}^{\prime}(R)-\left(k_{1}^{2}-k_{2}^{2}\right) f_{s}^{\prime}(R)}{\rho \omega^{2}\left(k_{1}^{2}-k_{2}^{2}\right) R}(\widehat{\mathbf{I}}, \widehat{\mathbf{R}} \widehat{\mathbf{R}}) \\
& +\frac{f_{s}(R)}{\mu} \widetilde{\mathbf{I}}_{3}, \\
\mathbf{E}_{12}\left(\mathbf{r}, \mathbf{r}^{\prime}\right)= & -\frac{\gamma k_{p}^{2}\left(f_{1}^{\prime}(R)-f_{2}^{\prime}(R)\right)}{\rho \omega^{2}\left(k_{1}^{2}-k_{2}^{2}\right)} \widehat{\mathbf{R}}, \\
\mathbf{E}_{21}\left(\mathbf{r}, \mathbf{r}^{\prime}\right)= & -\frac{q \kappa \eta}{\gamma} \mathbf{E}_{12}\left(\mathbf{r}, \mathbf{r}^{\prime}\right), \\
E_{22}\left(\mathbf{r}, \mathbf{r}^{\prime}\right)= & -\frac{\left(k_{p}^{2}-k_{1}^{2}\right) f_{1}(R)-\left(k_{p}^{2}-k_{2}^{2}\right) f_{2}(R)}{k_{1}^{2}-k_{2}^{2}} .
\end{aligned}
$$

Since

$$
\lim _{\varepsilon \rightarrow 0+} k_{1}^{2}=k_{p}^{2}
$$

and

$$
\lim _{\varepsilon \rightarrow 0+} k_{2}^{2}=q=i \frac{\omega}{k}
$$

we can easily deduce that

$$
\lim _{\varepsilon \rightarrow 0+} \widetilde{\mathbf{E}}_{11}\left(\mathbf{r}, \mathbf{r}^{\prime}\right)=\widetilde{\Gamma}\left(\mathbf{r}, \mathbf{r}^{\prime}\right)
$$

where

$$
\begin{aligned}
\widetilde{\boldsymbol{\Gamma}}\left(\mathbf{r}, \mathbf{r}^{\prime}\right)= & -\frac{f_{p}^{\prime \prime}(R)-f_{s}^{\prime \prime}(R)}{\rho \omega^{2}} \widehat{\mathbf{R}} \widehat{\mathbf{R}} \\
& -\frac{f_{p}^{\prime}(R)-f_{s}^{\prime}(R)}{\rho \omega^{2} R}\left(\widetilde{\mathbf{I}}_{3}-\widehat{\mathbf{R}} \widehat{\mathbf{R}}\right)+\frac{f_{s}(R)}{\mu} \widetilde{\mathbf{I}}_{3}
\end{aligned}
$$

is the fundamental dyadic of classical elasticity $[4,10]$. 
Similarly, for the dyadic of the fundamental stresses (38) we obtain

$$
\begin{aligned}
& \widetilde{\mathbf{E}}_{11}^{t}\left(\mathbf{r}, \mathbf{r}^{\prime}\right)=\frac{6}{k_{s}^{2} R^{3}}\left[\left(1-i k_{1} R\right) \frac{k_{1}^{2}-(1+\varepsilon) q}{k_{1}^{2}-k_{2}^{2}} f_{1}(R)\right. \\
& \left.-\left(1-i k_{2} R\right) \frac{k_{2}^{2}-(1+\varepsilon) q}{k_{1}^{2}-k_{2}^{2}} f_{2}(R)-\left(1-i k_{s} R\right) f_{s}(R)\right] \\
& \times\left[\left(\hat{\mathbf{n}}^{\prime} \cdot \widehat{\mathbf{R}}\right)\left(\widetilde{\mathbf{I}}_{3}-5 \widehat{\mathbf{R}} \widehat{\mathbf{R}}\right)+\hat{\mathbf{n}}^{\prime} \widehat{\mathbf{R}}+\widehat{\mathbf{R}}^{\prime} \hat{\mathbf{n}}^{\prime}\right] \\
& -\frac{\left(k_{1}^{2}-q\right) f_{1}(R)-\left(k_{2}^{2}-q\right) f_{2}(R)}{(\lambda+2 \mu)\left(k_{1}^{2}-k_{2}^{2}\right) R}\left[2 \mu\left(\hat{\mathbf{n}}^{\prime} \cdot \mathbf{R}\right)\left(\widetilde{\mathbf{I}}_{3}-6 \widehat{\mathbf{R}} \widehat{\mathbf{R}}\right)\right. \\
& \left.+2 \mu\left(\hat{\mathbf{n}}^{\prime} \widehat{\mathbf{R}}+\widehat{\mathbf{R}} \hat{\mathbf{n}}^{\prime}\right)-\lambda \hat{\mathbf{n}}^{\prime} \widehat{\mathbf{R}}\right] \\
& -\frac{\varepsilon q}{\left(k_{1}^{2}-k_{2}^{2}\right) R}\left[\left(1-i k_{1} R\right) f_{1}(R)-\left(1-i k_{2} R\right) f_{2}(R)\right] \hat{\mathbf{n}}^{\prime} \widehat{\mathbf{R}} \\
& -\frac{i k_{1}\left(k_{1}^{2}-q\right) f_{1}(R)-i k_{2}\left(k_{2}^{2}-q\right) f_{2}(R)}{k_{1}^{2}-k_{2}^{2}} \hat{\mathbf{n}}^{\prime} \cdot \frac{\lambda \widetilde{\mathbf{I}}_{3}+2 \mu \widehat{\mathbf{R}} \widehat{\mathbf{R}}}{\lambda+2 \mu} \widehat{\mathbf{R}} \\
& +\frac{f_{s}(R)}{R}\left[3\left(\hat{\mathbf{n}}^{\prime} \cdot \widehat{\mathbf{R}}\right)\left(\widetilde{\mathbf{I}}_{3}-4 \widehat{\mathbf{R}} \widehat{\mathbf{R}}\right)+3 \widehat{\mathbf{R}} \hat{\mathbf{n}}^{\prime}+2 \hat{\mathbf{n}}^{\prime} \widehat{\mathbf{R}}\right] \\
& -i k_{s} f_{s}(R)\left[\left(\hat{\mathbf{n}}^{\prime} \cdot \widehat{\mathbf{R}}\right)\left(\widetilde{\mathbf{I}}_{3}-2 \widehat{\mathbf{R}} \widehat{\mathbf{R}}\right)+\widehat{\mathbf{R}} \hat{\mathbf{n}}^{\prime}\right], \\
& \mathbf{E}_{12}^{t}\left(\mathbf{r}, \mathbf{r}^{\prime}\right)=\frac{2 q \varepsilon \mu}{\gamma\left(k_{1}^{2}-k_{2}^{2}\right) R^{2}}\left[\left(1-i k_{1} R\right) f_{1}(R)-\left(1-i k_{2} R\right) f_{2}(R)\right] \hat{\mathbf{n}}^{\prime} \cdot\left(\widetilde{\mathbf{I}}_{3}-3 \widehat{\mathbf{R}} \widehat{\mathbf{R}}\right) \\
& -\frac{2 q \varepsilon \mu}{\gamma\left(k_{1}^{2}-k_{2}^{2}\right)}\left(k_{1}^{2} f_{1}(R)-k_{2}^{2} f_{2}(R)\right) \hat{\mathbf{n}}^{\prime} \cdot\left(\widetilde{\mathbf{I}}_{3}-\widehat{\mathbf{R}} \widehat{\mathbf{R}}\right) \\
& +\frac{q \varepsilon \mu k_{s}^{2}}{\gamma\left(k_{1}^{2}-k_{2}^{2}\right)}\left(f_{1}(R)-f_{2}(R)\right) \hat{\mathbf{n}}^{\prime}, \\
& \mathbf{E}_{21}^{t}\left(\mathbf{r}, \mathbf{r}^{\prime}\right)=-\frac{\gamma}{(\lambda+2 \mu)\left(k_{1}^{2}-k_{2}^{2}\right) R^{2}}\left[\left(1-i k_{1} R\right) f_{1}(R)-\left(1-i k_{2} R\right) f_{2}(R)\right] \hat{\mathbf{n}}^{\prime} \cdot\left(\widetilde{\mathbf{I}}_{3}-3 \widehat{\mathbf{R}} \widehat{\mathbf{R}}\right) \\
& -\frac{\gamma\left(k_{1}^{2} f_{1}(R)-k_{2}^{2} f_{2}(R)\right)}{(\lambda+2 \mu)\left(k_{1}^{2}-k_{2}^{2}\right)} \hat{\mathbf{n}}^{\prime} \cdot \widehat{\mathbf{R}} \widehat{\mathbf{R}}
\end{aligned}
$$

and

$$
E_{22}^{t}\left(\mathbf{r}, \mathbf{r}^{\prime}\right)=\frac{\left(k_{1}^{2}-k_{p}^{2}\right)\left(1-i k_{1} R\right) f_{1}(R)-\left(k_{2}^{2}-k_{p}^{2}\right)\left(1-i k_{2} R\right) f_{2}(R)}{\left(k_{1}^{2}-k_{2}^{2}\right) R} \hat{\mathbf{n}}^{\prime} \cdot \widehat{\mathbf{R}}
$$

\section{REFERENCES}

[1] F. V. Atkinson, On Sommerfeld's radiation condition, Philos. Mag. Series 7, 40, 645-651 (1949)

[2] M. Biot, Thermoelasticity and irreversible thermodynamics, J. Appl. Phys. 27, 240-253 (1956)

[3] F. Cakoni and G. Dassios, The coated thermoelastic body within a low-frequency elastodynamic field, Internat. J. Engrg. Sci. 36, 1815-1838 (1998)

[4] G. Dassios, The Atkinson-Wilcox expansion theorem for elastic waves, Quart. Appl. Math. 46, 285-299 (1988)

[5] G. Dassios and V. Kostopoulos, The scattering amplitudes and cross sections in the theory of thermoelasticity, SIAM J. Appl. Math. 48, 79-98 (1988). Errata: SIAM J. Appl. Math. 49, 12831284 (1989)

[6] G. Dassios and V. Kostopoulos, On Rayleigh expansions in thermoelastic scattering, SIAM J. Appl. Math. 50, 1300-1324 (1990) 
[7] G. Dassios and V. Kostopoulos, Thermoelastic Rayleigh scattering by a rigid ellipsoid, Mat. Aplic. Comput. 9, 153-173 (1990)

[8] G. Dassios and V. Kostopoulos, Scattering of elastic waves by a small thermoelastic body, Internat. J. Engrg. Sci. 32, 1593-1603 (1994)

[9] S. N. Karp, A convergent "farfield" expansion for two-dimensional radiation functions, Comm. Pure Appl. Math. 14, 427-434 (1961)

[10] V. D. Kupradze, Three-dimensional Problems of the Mathematical Theory of Elasticity and Thermoelasticity, North-Holland, Amsterdam, 1979

[11] J. C. Maxwell, Treatise on Electricity and Magnetism, 2 vols., third edition, Dover, New York, 1954.

[12] A. Sommerfeld, Die Greensche Funktion der Schwingungsgleichung, Jahr. Der. Deut. Math. Ver. 21, 309-353 (1912)

[13] V. Twersky, Rayleigh scattering, Applied Optics 3, 1150-1162 (1964)

[14] C. H. Wilcox, A generalization of theorems of Rellich and Atkinson, Proc. Amer. Math. Soc. 7, 271-276 (1956)

[15] C. H. Wilcox, An expansion theorem for electromagnetic fields, Comm. Pure Appl. Math. 9, 115-134 (1956) 